\title{
Computational multiscale modelling of heterogeneous material layers
}

\author{
C. B. Hirschberger; S. Ricker, P. Steinmann; N. Sukumar ${ }^{\S}$ \\ Manuscript as accepted for publication in Engineering Fracture Mechanics, 26 October 2008
}

\begin{abstract}
A computational homogenization procedure for a material layer that possesses an underlying heterogeneous microstructure is introduced within the framework of finite deformations. The macroscopic material properties of the material layer are obtained from multiscale considerations. At the macro level, the layer is resolved as a cohesive interface situated within a continuum, and its underlying microstructure along the interface is treated as a continuous representative volume element of given height. The scales are linked via homogenization with customized hybrid boundary conditions on this representative volume element, which account for the deformation modes along the interface. A nested numerical solution scheme is adopted to link the macro and micro scales. Numerical examples successfully display the capability of the proposed approach to solve macroscopic boundary value problems with an evaluation of the constitutive properties of the material layer based on its micro-constitution.
\end{abstract}

\section{Introduction}

Material layers that transmit cohesive tractions occur in several engineering disciplines. Solder connections, adhesive bonding layers, laminated composite structures, building materials such as masonry, as well as geomaterials are some notable examples. Two are illustrated in Figure 1. In most cases, the material in the connecting layer is significantly weaker than the surrounding bulk material and therefore the deformation will

${ }^{*}$ c.b.hirschberger@tue.nl, Department of Mechanical Engineering, Eindhoven University of Technology, P.O. Box 513, 5600 MB Eindhoven, The Netherlands (formerly at University of Kaiserslautern, Germany) †sricker@rhrk.uni-kl.de, Department of Mechanical and Process Engineering, University of Kaiserslautern, PO Box 3049, 67753 Kaiserslautern, Germany

*steinmann@ltm.uni-erlangen.de, Department of Mechanical Engineering, Friedrich-Alexander University of Erlangen-Nuremberg, Egerlandstraße 5, 91058 Erlangen, Germany

${ }^{\S}$ nsukumar@ucdavis.edu, Department of Civil and Environmental Engineering, University of California at Davis, One Shields Avenue, Davis, CA 95616, USA 
(a)

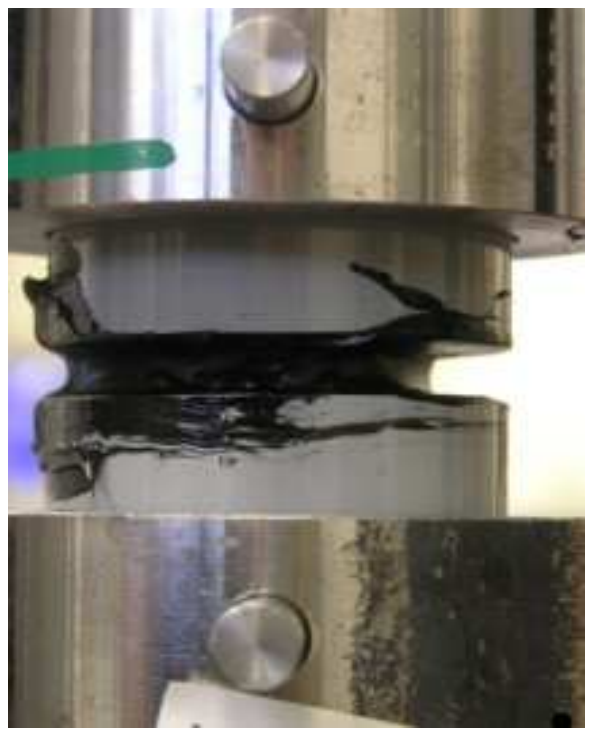

(b)

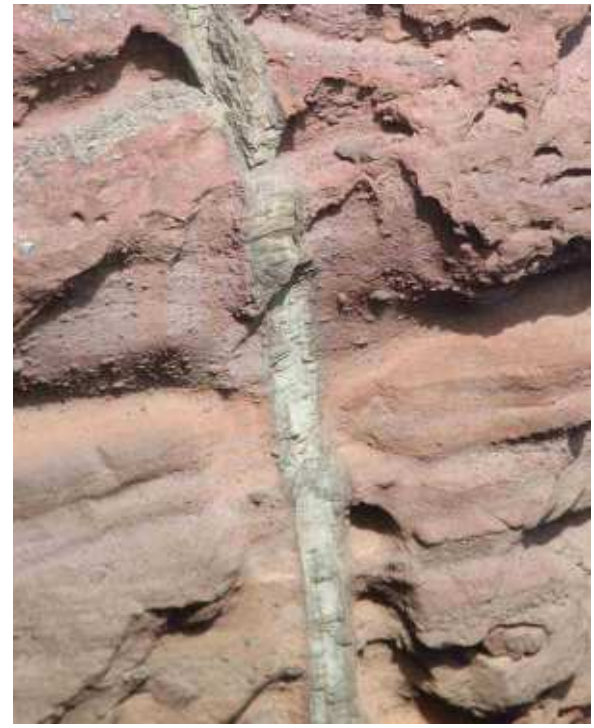

Figure 1: (a) Adhesive bonding of two solid substrates with a polymeric glue: circular uni-axial tension specimen with incompletely cured polyurethane layer (courtesy of Gunnar Possart). (b) Material layer within geological bulk material of different properties.

be strongly confined to this layer. If the material layer is composed of a heterogeneous microstructure, the geometric and material properties of that will crucially govern the global behaviour. Such heterogeneities can for instance appear as voids, micro cracks, or inclusions, and can be found in fibre-reinforced materials (e. g. , metal-polymer matrix, concrete), or in natural materials (e.g. , geological conglomerates). Homogenization approaches as pioneered by Hill $[4,5]$ provide an appropriate framework to relate the mechanical behaviour within the different spatial scales of observation. The key issue of the current contribution is to account for this microstructure of the material layer in an appropriate way. Beyond existing approaches, which are achieved for instance by asymptotic homogenization $[15,16]$, we particularly aim to propose a computational multiscale framework that is suitable for nonlinear multiscale finite-element simulations in the spirit of $\mathrm{FE}^{2}$.

Within a multiscale consideration, on the macro scale the material layer is treated as a cohesive interface situated within a continuum. The governing quantities in this cohesive interface, i. e. the displacement jump (or rather separation) and the cohesive tractions, are related based on the underlying microstructure rather than employing an a priori constitutive assumption, coined as a cohesive traction-separation law. On the micro scale, representative volume elements (RVE) along the material layer advocate the heterogeneous microstructure, as illustrated in Figure 2. Their height is directly given by the thickness of the material layer. For the concept of representative volume elements the reader is for instance referred to References [5, 28]. The micro-macro transition between the RVE and the interface is achieved based upon the averaging of the gov- 


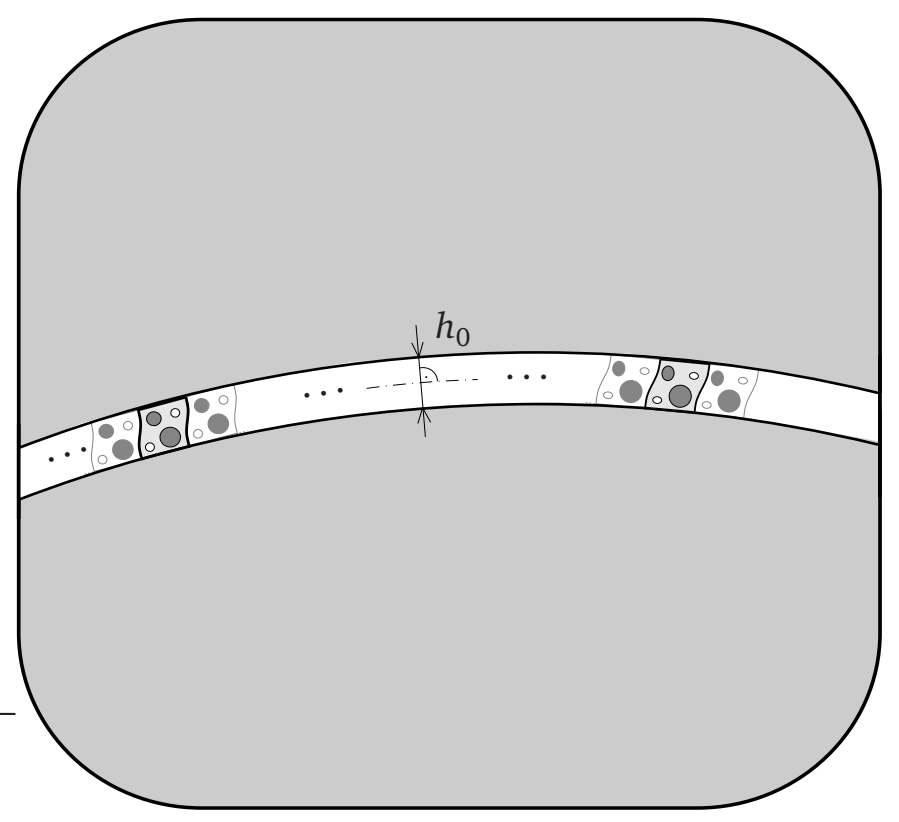

Figure 2: Heterogeneous material layer, which is shown over-sized for the sake of visibility, situated within a macro bulk material with sketches of RVEs that are locally periodic, but may vary along the material layer.

erning kinematic, stress and energetic quantities over the respective underlying RVE. The boundary conditions stemming from the cohesive interface at the macro level imposed on the RVE must be chosen consistently_on the one hand, they need to fulfil the Hill condition [5], which ensures the equivalence of the macro and the micro response, whereas on the other hand, the boundary conditions shall account for the interface geometry and capture the occurring mixed-mode (shear and tension) deformation modes.

The homogenization approach is numerically implemented within a computational homogenization along the lines of References [9, 10, 11, 20, 22, 21, 25, 24]. Within a geometrically nonlinear finite-element framework, we straightforwardly model the material layer by means of cohesive interface elements situated between the adjacent bulk finite elements. With these elements, the finite-element formulation which can be found in References [1, 18, 23, 29, 30, 34, 35, 37], the constitutive relation consists by a cohesive traction-separation law, which has traditionally been treated by a priori assumptions, as they were proposed by $\mathrm{Xu}$ and Needleman [27, 38]. Instead of using such constitutive assumption, we obtain the material response from computational homogenization. To this end, the solution of a micro scale boundary value problem is invoked at each integration point of each interface elements. Thereby upon application of customized hybrid boundary conditions stemming from the interface, the macroscopic constitutive behaviour is extracted at the RVE boundaries towards the bulk. The representative volume element is modelled as a nonlinear finite-element boundary value problem, which is subjected to the deformation induced by the interface element on 
the macro scale. The macroscopic traction and the constitutive tangent operator for a Newton-Raphson solution scheme are extracted from the micro problem. In this way, a fully nested iterative multiscale solution for a bulk including a material layer accounting for the micro-heterogeneous properties of the latter is accomplished.

The current paper extends the multiscale approach of Matous et al. [17] to the general case of finite deformations. Beyond both the latter contribution and that of Larsson and Zhang [14], not only the cohesive behaviour of the microscopically heterogeneous material layer shall be considered, but rather we are interested in solving macroscopic boundary value problems involving this material layer. To this end, emphasis is placed on a multiscale framework, that utilizes computational homogenization. If the intrinsic microstructure is negligibly small such that no size effects occur, as is the case in the current paper, we restrict ourselves to a classical (Boltzmann) continuum within the representative volume element. In contrast, if the size effect of the intrinsic microstructure is significant, the microstructure can be modelled as a micromorphic continuum, which is pursued in Reference [7].

\subsection{Outline and Notation}

The remainder of the paper is structured as follows: In Section 2, we present the continuum mechanics framework on the macro scale with the material layer treated as a cohesive interface. In Section 3, the governing equations for the representative volume element that represents the underlying microstructure, are presented. Once both the macro and the micro level descriptions are present, the micro-macro transition based on the homogenization of the decisive micro quantities is examined in Section 4. Section 5 provides the numerical framework of the computational homogenization. Numerical examples in Section 6 exhibit the main features of the proposed approach, and finally some concluding remarks are mentioned in Section 7. For the sake of distinction and clarity, the quantities on the macro scale are denoted by an over-bar $(\bar{\cdot})$, whereas all other quantities refer to the micro scale.

\section{Material layer represented by an interface at the macro level}

On the macro scale we consider a body $\overline{\mathscr{B}}_{0}$ that consists of a bulk that is separated by a thin material layer of significantly different properties. We treat this layer as an interface, $\bar{\Gamma}_{0}$, as illustrated in Figure 3. On the interface we define the unit normal vector $\bar{N}$ as

$$
\bar{N}(\bar{X})=-\bar{N}^{+}(\bar{X})=+\bar{N}^{-}(\bar{X}), \quad \forall \bar{X} \in \bar{\Gamma}_{0} .
$$

Thereby $\bar{N}^{+}(\bar{X})$ is the outward normal on the positive part $\overline{\mathscr{B}}_{0}^{+}$and $\bar{N}^{-}(\bar{X})$ on the negative part $\overline{\mathscr{B}}_{0}^{-}$, respectively. In the following, we introduce the governing continuum mechanics framework that defines a general boundary value problem for the macro level involving this interface. 

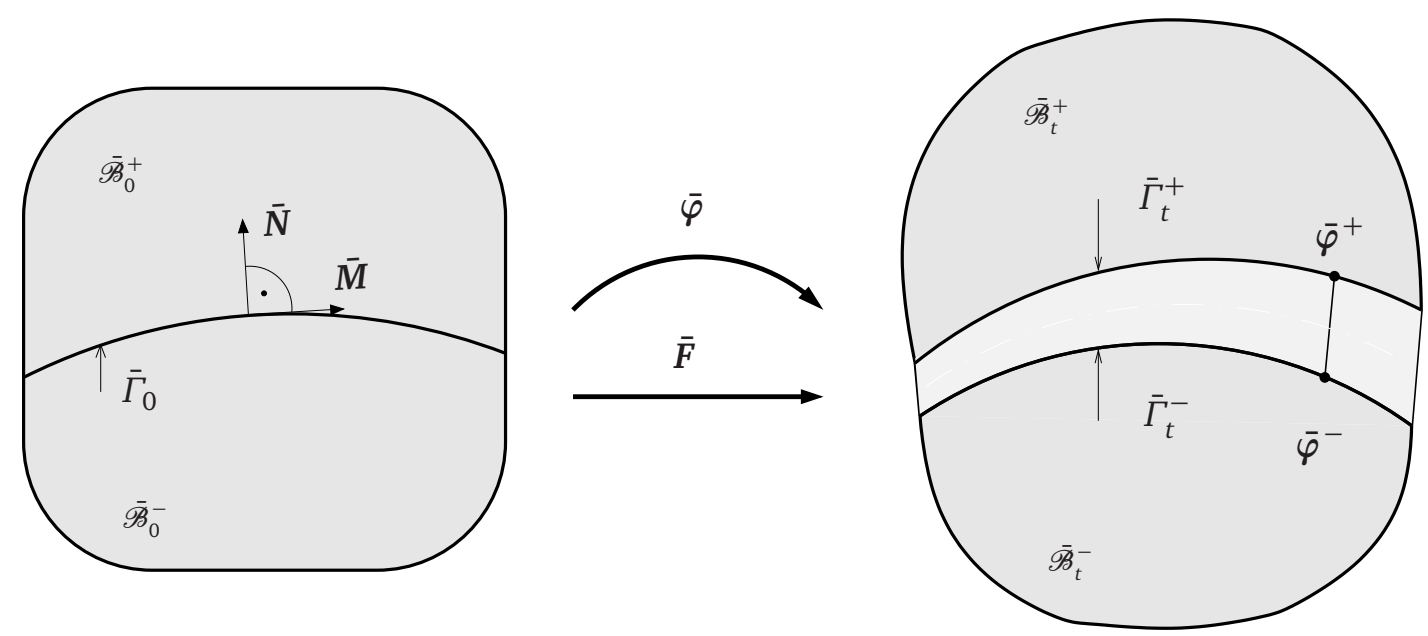

Figure 3: Interface geometry and deformation maps from the material configuration $\bar{\Gamma}_{0}$ to the spatial configuration $\bar{\Gamma}_{t}$.

\subsection{Deformation}

The deformation in the bulk is described via the deformation map $\bar{x}=\bar{\varphi}(\bar{X})$ and its gradient $\overline{\boldsymbol{F}}:=\nabla_{\bar{X}} \bar{\varphi}$ for all material points $\bar{X} \in \overline{\mathscr{B}}_{0}^{+} \cup \overline{\mathscr{B}}_{0}^{-} \backslash \bar{\Gamma}_{0}$.

The deformation jump or separation between the opposite spatial edges of the interface,

$$
\llbracket \bar{\varphi} \rrbracket(\bar{X}):=\bar{\varphi}^{+}(\bar{X})-\bar{\varphi}^{-}(\bar{X}) \quad \forall \bar{X} \in \bar{\Gamma}_{0},
$$

acts as the primary deformation quantity of the interface. The vectorial representation at this point incorporates a loss of information compared to the full deformation tensor. Nevertheless, for the considered material layer this assumption is fully sufficient, since its initial height $h_{0}$ is much smaller than the total extension of the bulk.

\subsection{Equilibrium}

For the material body $\overline{\mathscr{B}}_{0}$ to be in equilibrium the balance of momentum for the bulk $\overline{\mathscr{B}}_{0} \backslash \bar{\Gamma}_{0}$ and the equilibrium relations for the cohesive interface $\bar{\Gamma}_{0}$ must be fulfilled. The balance of momentum for the bulk reads

$$
\operatorname{Div} \overline{\boldsymbol{P}}=-\boldsymbol{b}_{0} \quad \text { in } \overline{\mathscr{B}}_{0} \backslash \bar{\Gamma}_{0},
$$

in terms of the Piola stress $\overline{\boldsymbol{P}}$. The corresponding Neumann and Dirichlet boundary conditions prescribe the spatial traction $\overline{\boldsymbol{t}}_{0}$ with respect to material reference or the deformation map $\bar{\varphi}$ on the respective part of the boundary:

$$
\overline{\boldsymbol{P}} \cdot \overline{\boldsymbol{N}}=: \overline{\boldsymbol{t}}_{0}^{\text {pre }} \quad \text { on } \partial \overline{\mathscr{B}}_{0}^{P}, \quad \bar{\varphi}=: \bar{\varphi}^{\text {pre }} \text { on } \partial \overline{\mathscr{B}}_{0}^{\varphi} .
$$


Across the interface, cohesive tractions are transmitted. The additional equilibrium condition concerning the interface,

$$
\overline{\boldsymbol{t}}_{0}^{+}+\overline{\boldsymbol{t}}_{0}^{-}=\mathbf{0}
$$

together with the Cauchy theorem entails a relation for the jump of the Piola stress, $\llbracket \overline{\boldsymbol{P}} \rrbracket$, and for its average $\{\overline{\boldsymbol{P}}\}$ across the discontinuity:

$$
\llbracket \overline{\mathbf{P}} \rrbracket \cdot \overline{\boldsymbol{N}}=\mathbf{0}, \quad\{\overline{\boldsymbol{P}}\} \cdot \overline{\boldsymbol{N}}=\overline{\boldsymbol{t}}_{0} \quad \text { on } \bar{\Gamma}_{0} .
$$

The weak formulation of the balance relations (3)-(6) renders the virtual work statement, which requires the sum of the internal contributions of both the bulk and the interface to equal the external virtual work:

$$
\int_{\mathscr{\mathscr { B }}_{0} \backslash \bar{\Gamma}_{0}} \overline{\boldsymbol{P}}: \delta \overline{\boldsymbol{F}} \mathrm{d} V+\int_{\bar{\Gamma}_{0}} \overline{\boldsymbol{t}}_{0} \cdot \llbracket \delta \bar{\varphi} \rrbracket \mathrm{d} A=\int_{\mathscr{\mathscr { B }}_{0}} \overline{\boldsymbol{b}}_{0} \cdot \delta \bar{\varphi} \mathrm{d} V+\int_{\partial \overline{\mathscr{B}}_{0}^{p}} \overline{\boldsymbol{t}}_{0}^{\text {pre }} \cdot \delta \bar{\varphi} \mathrm{d} A .
$$

The relation between the stress and the deformation measures is supplied via a constitutive relation.

\subsection{Constitutive framework}

For the surrounding bulk, we avail ourselves of a hyperelastic constitutive formulation which is stated a priori, for instance a neo-Hooke ansatz. Thus the Piola stress is evaluated from the stored-energy density as $\overline{\boldsymbol{P}}=\mathrm{D}_{\overline{\mathrm{F}}} \overline{\mathscr{W}}_{0}$.

The traction $\overline{\boldsymbol{t}}_{0}$ transmitted across the cohesive interface is energetically conjugate to the separation $\llbracket \bar{\varphi} \rrbracket$ in a hyperelastic format. Within an entirely reversible isothermal constitutive framework, it is a function of the interface separation, i.e. $\overline{\boldsymbol{t}}_{0}(\llbracket \bar{\varphi} \rrbracket)$. Our objective is to find such a relation based on the underlying microstructure using a multiscale approach. Particularly, in the context of a numerical finite-element simulation utilizing a Newton-Raphson procedure, we are moreover interested in the tangent operator $\bar{A}$ in an incremental traction-separation law,

$$
\delta \bar{t}_{0}=\bar{A} \cdot \llbracket \delta \bar{\varphi} \rrbracket, \quad \bar{A}:=\mathrm{D}_{\llbracket \bar{\varphi} \rrbracket} \bar{t}_{0} .
$$

Towards a multiscale framework we will next present a formulation for the underlying microstructure and thereafter bridge the two scales by means of homogenization.

\section{Representative volume element at the micro level}

Within the proposed multiscale approach, we now consider the modelling of the underlying heterogeneous microstructure. As was illustrated in Figure 2, representative volume elements are used to model statistically representative portions of the material layer. To match them with the interface geometry, we align these volume elements with 


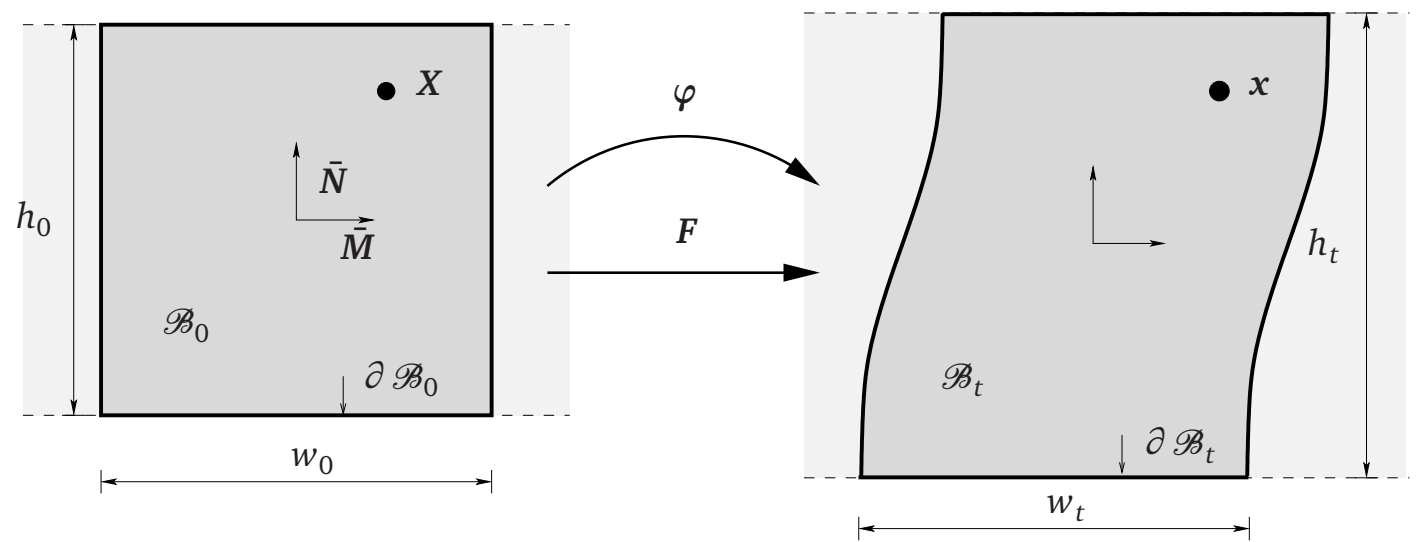

Figure 4: RVE geometry and deformation maps from the material configuration $\mathscr{B}_{0}$ to the spatial configuration $\mathscr{B}_{t}$.

the interfacial plane and limit its dimension out of plane by the initial height $h_{0}$ of the material layer. Thereby the dimension of the RVE in plane must be chosen sufficiently large to make the element representative, yet small enough compared to the in plane dimension of the layer to exclude boundary effects. In the two-dimensional setting pursued here, such element has an initial width $w_{0}$ and thus a material volume (or rather area) of $V_{0}=w_{0} h_{0}$.

Any appropriate mechanical framework could be employed on the RVE level, such as a continuum (either in a standard, a higher-order or a higher-grade formulation), discrete particles to account for granular media, molecular dynamics, or atomistics, just to mention a few. However, in this article we restrict ourselves to a standard (or rather Boltzmann) continuum, whereas a further extension to a micromorphic RVE accounting for size effects induced by a significant intrinsic microstructure can be found in Reference [7]. In order to clarify the notation at the micro level, we will in the following briefly review the governing equations stating a boundary value problem on the RVE. Based upon this geometrically nonlinear framework, the connections with the macro problem will be treated in Section 4.

\subsection{Deformation}

The finite deformation, also illustrated in Figure 4, is described through the deformation map $\varphi$ and the deformation gradient $F$ :

$$
x=\varphi(X), \quad F(X):=\nabla_{X} \varphi(X) \quad \forall X \in \partial \mathscr{B}_{0} .
$$

The placement $X$ can be chosen with respect to any basis; however for practical reasons corresponding to the micro-macro transition, the origin shall be placed in the geometric centre of the RVE. 


\subsection{Equilibrium}

The representative volume element is in equilibrium if the balance of momentum for the static case,

$$
\operatorname{Div} \boldsymbol{P}=\mathbf{0} \quad \text { in } \mathscr{B}_{0},
$$

is fulfilled under the supplied Neumann and Dirichlet boundary conditions:

$$
\boldsymbol{P} \cdot \boldsymbol{N}=: \boldsymbol{t}_{0}^{\mathrm{pre}} \quad \text { on } \partial \mathscr{B}_{0}^{P}, \quad \varphi=: \varphi^{\text {pre }} \text { on } \partial \mathscr{B}_{0}^{\varphi} .
$$

Thereby at a particular part of the boundary, either the spatial traction $\boldsymbol{t}_{0}=\boldsymbol{P} \cdot \boldsymbol{N}$ or the deformation $\varphi$ may be prescribed, with $\partial \mathscr{B}_{0}^{P} \cap \partial \mathscr{B}_{0}^{\varphi}=\emptyset$.

At the micro level, the influence of the body force is neglected, as suggested for instance by [21]. This choice proves convenient in view of the homogenization, which utilizes only quantities on the RVE boundary. With this assumption, the corresponding virtual work statement at the micro level reads:

$$
\int_{\mathscr{B}_{0}} \boldsymbol{P}: \delta \boldsymbol{F} \mathrm{d} V=\int_{\partial \mathscr{B}_{0}} \boldsymbol{t}_{0} \cdot \delta \varphi \mathrm{d} A .
$$

\subsection{Constitutive framework}

Any appropriate constitutive formulation could be incorporated. However, for the sake of clarity of exposition, we avail ourselves of a straightforward hyperelastic format for the stored-energy density.

\subsection{Boundary value problem}

The representative volume element is subjected to boundary conditions that stem from the interfacial traction and separation at the macro level. The necessary relations connecting the two scales consistently with respect to the geometry of the material layer will be addressed in the following section.

\section{Micro-macro transition}

The proposed homogenization approach is based on the averaging of the governing quantities over the volume of the RVE as proposed by Hill $[4,5]$. First, we recall the volume averages of the deformation gradient, the stress, and the virtual work over the RVE, as they are well-known from the literature. Then, these RVE averages are related to the governing quantities in the interface. Boundary conditions on the RVE finalize a consistent scale transition. 


\subsection{Averages of micro quantities over the RVE}

The average of the deformation gradient $\boldsymbol{F}$ over the volume of the RVE is given as

$$
\langle\boldsymbol{F}\rangle=\frac{1}{V_{0}} \int_{\mathscr{B}_{0}} \boldsymbol{F} \mathrm{d} V=\frac{1}{V_{0}} \int_{\partial \mathscr{B}_{0}} \varphi \otimes N \mathrm{~d} A .
$$

The volume average of the Piola stress $\boldsymbol{P}$ in the RVE,

$$
\langle\boldsymbol{P}\rangle=\frac{1}{V_{0}} \int_{\mathscr{B}_{0}} \boldsymbol{P} \mathrm{d} V=\frac{1}{V_{0}} \int_{\partial \mathscr{B}_{0}} \boldsymbol{t}_{0} \otimes X \mathrm{~d} A,
$$

is required in view of the macroscopic traction vector $\overline{\boldsymbol{t}}_{0}$ in the interface given in (6) 2 . Finally, based on (12) the average of the virtual work in the RVE reads

$$
\langle\boldsymbol{P}: \delta \boldsymbol{F}\rangle=\frac{1}{V_{0}} \int_{\mathscr{B}_{0}} \boldsymbol{P}: \delta \boldsymbol{F} \mathrm{d} V=\frac{1}{V_{0}} \int_{\partial \mathscr{B}_{0}} \delta \varphi \cdot \boldsymbol{t}_{0} \mathrm{~d} A .
$$

Thereby, as already mentioned in Section 3.1, we assume without loss of generality that the origin of the coordinate system is placed in the geometric centre of the RVE. The following canonical auxiliary relations $[2,21]$,

$$
\boldsymbol{F}=\operatorname{Div}(\boldsymbol{\varphi} \otimes I), \quad \quad \boldsymbol{P}^{\mathrm{t}}=\operatorname{Div}(\boldsymbol{X} \otimes \boldsymbol{P}), \quad \boldsymbol{P}: \boldsymbol{F}=\operatorname{Div}(\boldsymbol{\varphi} \cdot \boldsymbol{P}),
$$

are utilized to convert the averaging theorems from volume to surface integrals. For the latter two conversions, the equilibrium in omission of body forces was used, as for instance also documented in References [10].

\subsection{Micro-macro transition}

In order to accomplish a consistent transition between the micro and the macro level, the averaged RVE quantities need to be related to the interface quantities. Therefore, the deformation, the traction as well as the virtual energy need to be equivalent on both scales.

\subsubsection{Deformation}

Upon the consideration of the initial height $h_{0}$ of the material layer, the averaged deformation gradient (13) is linked to the interface kinematics on the macro level as follows: Since the governing kinematic quantity on the RVE level is given by the homogenized deformation gradient, which is tensor of second order, it is desirable to find a secondorder tensor to represent the macro interface deformation as well. Therefore we avail 
ourselves of a deformation tensor, which was first proposed in the context of localized plasticity, (see, for instance References [12, 13, 31, 32]):

$$
\overline{\boldsymbol{F}}:=I+\frac{1}{h_{0}} \llbracket \bar{\varphi} \rrbracket \otimes \bar{N} .
$$

Instead of an artificial scaling parameter, which in those approaches is used to achieve regularization, here indeed the initial height $h_{0}$ enters this deformation tensor. Clearly, this measure resolves the information given by the macro separation as follows:

$$
\bar{F}=\bar{M} \otimes \bar{M}+\left[\frac{1}{h_{0}} \llbracket \bar{\varphi} \rrbracket \cdot \bar{M}\right] \bar{M} \otimes \bar{N}+\left[1+\frac{1}{h_{0}} \llbracket \bar{\varphi} \rrbracket \cdot \bar{N}\right] \bar{N} \otimes \bar{N},
$$

or translated into a straightforward matrix notation with respect to the orthonormal basis $(\bar{M}, \bar{N})$ :

$$
\overline{\boldsymbol{F}} \hat{=}\left[\begin{array}{rr}
1 & \llbracket \bar{\varphi}_{\bar{M}} \rrbracket / h_{0} \\
0 & 1+\llbracket \bar{\varphi}_{\bar{N}} \rrbracket / h_{0}
\end{array}\right]
$$

With this macro assumption at hand, we can relate the macro deformation to the RVE average deformation gradient as

$$
I+\frac{1}{h_{0}} \llbracket \bar{\varphi} \rrbracket \otimes \bar{N} \equiv\langle F\rangle .
$$

Although it obeys the restriction that $\bar{M} \cdot \bar{F} \cdot \bar{M}=1$, it involves all the information that is contained in the vectorial representation of the interface separation $\llbracket \bar{\varphi} \rrbracket$. This assumption yields a somewhat rigorous restriction on the deformation of the RVE, as we will examine later on.

\subsubsection{Traction}

The traction $\overline{\boldsymbol{t}}_{0}$ in the interface is related to the averaged Piola stress (14) in the underlying RVE based on the Cauchy theorem (6) ${ }_{2}$ :

$$
\overline{\boldsymbol{t}}_{0} \equiv\langle\boldsymbol{P}\rangle \cdot \overline{\boldsymbol{N}} .
$$

assuming the average RVE Piola stress to be equivalent to the average across the interface of the Piola stress on the macro scale $\langle\boldsymbol{P}\rangle \equiv\{\overline{\boldsymbol{P}}\}$.

\subsubsection{Virtual work}

The Hill condition requires the virtual work performed in the interface to be equivalent to the average of the virtual work performed within the representative volume element. The RVE virtual work density, $\boldsymbol{P}: \delta \boldsymbol{F}$, acts within a continuum element $\mathrm{d} V$ and the interface virtual work density is referred to a surface element $\mathrm{d} A$. Due to their different 


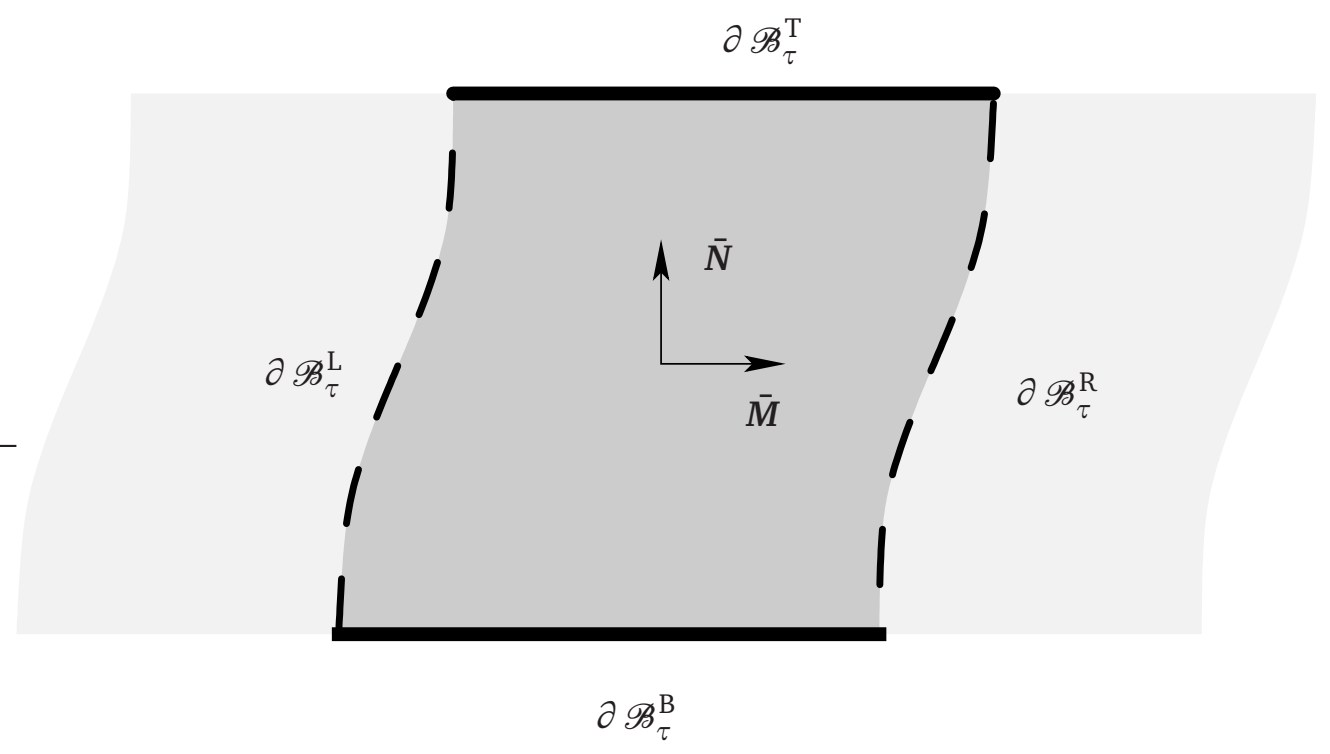

Figure 5: Hybrid boundary conditions on the RVE: prescribed deformation on $\partial \mathscr{B}_{\tau}^{\mathrm{T}}$ and $\partial \mathscr{B}_{\tau}^{\mathrm{B}}$; periodic deformation $\partial \mathscr{B}_{\tau}^{\mathrm{R}}$ and $\partial \mathscr{B}_{\tau}^{\mathrm{L}}, \tau \in\{0, t\}$.

dimension, the average of the virtual work in the underlying RVE, (15), needs to be scaled by the height $h_{0}$ of the material layer and we obtain

$$
\overline{\boldsymbol{t}}_{0} \cdot \llbracket \delta \bar{\varphi} \rrbracket \equiv h_{0}\langle\boldsymbol{P}: \delta \boldsymbol{F}\rangle .
$$

With the particular equivalences of the interfacial separation (17) and traction (21), with this condition the usual requirement in the form

$$
\langle\boldsymbol{P}\rangle:\langle\delta \boldsymbol{F}\rangle \equiv\langle\boldsymbol{P}: \delta \boldsymbol{F}\rangle .
$$

is retrieved. In this form the Hill condition requires the average of the virtual work performed in the RVE to equal the virtual work performed by the respective averages of the deformation gradient, (13), and the stress, (14), and was thus also referred to as the macro-homogeneity condition [2].

\subsection{Boundary conditions on the RVE}

The micro-macro transition is achieved by the choice of appropriate boundary conditions on the RVE. These are governed by the corresponding quantities in the interface and must fulfil the Hill condition (23) to be admissible. Generally the boundary conditions to impose on the RVE will depend on the deformation and the traction in the interface. However, we omit the rather tedious application of traction boundary conditions since we are aiming at a deformation- driven computational homogenization.

Due to the vectorial representation of the deformation jump within the material layer, only two deformation modes can occur in the interface, i.e. relative shear and normal 
tension/compression. Furthermore, the extension of the interface in tangential direction is by orders larger than its height, which gives rise to boundary conditions that are hybrid between linear displacement and periodic displacements and anti-periodic tractions on the RVE boundary, as depicted in Figure 5 and examined in the sequel.

\subsubsection{Choice of boundary conditions}

For the first part of the hybrid choice of boundary conditions, we fully prescribe the boundary conditions by means of the macro interface opening $\llbracket \bar{\varphi} \rrbracket$ on the top and the bottom boundaries of representative volume element, since these are conceptually aligned with the positive and negative edges of the cohesive interface, $\bar{\Gamma}_{0}^{+}$and $\bar{\Gamma}_{0}^{-}$, respectively. This displacement boundary condition is first expressed in terms of the proxy macro deformation tensor of (13), which is easily transferred into an expression in terms of the deformation jump only:

$$
\varphi(X)=\left[I+\frac{1}{h_{0}} \llbracket \bar{\varphi} \rrbracket \otimes \bar{N}\right] \cdot X=\left\{\begin{array}{cc}
X+\frac{1}{2} \llbracket \bar{\varphi} \rrbracket & \forall X \in \partial \mathscr{B}_{0}^{\mathrm{T}} \\
X-\frac{1}{2} \llbracket \bar{\varphi} \rrbracket & \forall X \in \partial \mathscr{B}_{0}^{\mathrm{B}}
\end{array} .\right.
$$

As a second ingredient of the hybrid boundary conditions, in tangential direction of the interface (or rather in-plane), we assume periodic deformation and anti-periodic traction boundary conditions

$$
\delta \varphi^{\mathrm{R}}-\delta \varphi^{\mathrm{L}}=\mathbf{0} \quad \boldsymbol{t}_{0}^{\mathrm{R}}+\boldsymbol{t}_{0}^{\mathrm{L}}=\mathbf{0}
$$

on the RVE, whereby the notations

$$
\begin{array}{lll}
\delta \varphi^{\mathrm{R}}:=\delta \varphi(X), & t_{0}^{\mathrm{R}}:=\boldsymbol{t}_{0}(X) & \forall X \in \partial \mathscr{B}_{0}^{\mathrm{R}} \\
\delta \varphi^{\mathrm{L}}:=\delta \varphi(X), & \boldsymbol{t}_{0}^{\mathrm{L}}:=\boldsymbol{t}_{0}(X) & \forall X \in \partial \mathscr{B}_{0}^{\mathrm{L}}
\end{array}
$$

are used. The straightforward proof of the admissibility of this choice of boundary conditions is examined in the following section.

The vectorial representation of both separation and traction in the interface restricts the deformation to two deformation modes: shearing tangential to the interface plane and tension out of the interface plane, which is also reflected by the deformation measure in (13). Therefore, with this model, it is not possible to account for in-plane tension within the RVE. However, the macro level does not sense this restriction and the lateral contraction along the interface is entirely controlled by the surrounding bulk in a natural manner.

\subsubsection{Admissibility of hybrid boundary conditions}

To show that this choice of boundary condition fulfil the Hill condition (23), the relation $\langle\boldsymbol{P}\rangle:\langle\delta \boldsymbol{F}\rangle=\langle\boldsymbol{P}:\langle\delta \boldsymbol{F}\rangle\rangle$ is used. The Hill condition holds if the following identity is fulfilled:

$$
h_{0}\langle\boldsymbol{P}: \delta \boldsymbol{F}\rangle-h_{0}\langle\boldsymbol{P}:\langle\delta \boldsymbol{F}\rangle\rangle \doteq 0
$$


For the proposed hybrid boundary conditions, this relation is both shown to be fulfilled for the prescribed deformation on the top and the bottom boundary of the RVE and for the periodic in-plane deformation.

To this end, the relation is transformed to the following:

$$
h_{0}\langle\boldsymbol{P}: \delta \boldsymbol{F}\rangle-h_{0}\langle\boldsymbol{P}:\langle\delta \boldsymbol{F}\rangle\rangle=\frac{1}{w_{0}} \int_{\partial \mathscr{B}_{0}} \boldsymbol{t}_{0} \cdot[\delta \varphi-\langle\delta \boldsymbol{F}\rangle \cdot \boldsymbol{X}] \mathrm{d} A_{0},
$$

For the prescribed displacement (24) the term in brackets directly vanishes and thus the entire integral becomes zero.

In a second step, the periodic boundary conditions (25) in-plane are shown to be admissible by regarding (29). Since for a macro deformation (24) affinely imposed on the RVE, the term $\langle\delta \boldsymbol{F}\rangle \cdot X$ is periodic to begin with, thus the fluctuation term $\delta \varphi-\langle\delta \boldsymbol{F}\rangle \cdot X$ proves periodic as well. Consequently the integral

$$
\frac{1}{w_{0}} \int_{\partial \mathscr{B}_{0}^{\mathrm{L}}} \boldsymbol{t}_{0} \cdot[\delta \varphi-\langle\delta \boldsymbol{F}\rangle \cdot \boldsymbol{X}] \mathrm{d} A+\frac{1}{w_{0}} \int_{\partial \mathscr{B}_{0}^{\mathrm{R}}} \boldsymbol{t}_{0} \cdot[\delta \varphi-\langle\delta \boldsymbol{F}\rangle \cdot \boldsymbol{X}] \mathrm{d} A=0
$$

vanishes over opposite periodic boundaries if the traction $t_{0}$ is anti-periodic, which itself follows from equilibrium. Due to their periodicity, the sum of the integrals over the opposite edges on the left and the right side $\mathscr{B}_{0}^{\mathrm{L}}$ and $\mathscr{B}_{0}^{\mathrm{R}}$ respectively, vanishes as described.

To gather all contributions of the hybrid boundary conditions, we build the sum of the particular parts of (29) and (30),

$$
\begin{gathered}
h_{0}\langle\boldsymbol{P}: \delta \boldsymbol{F}\rangle-h_{0}\langle\boldsymbol{P}:\langle\delta \boldsymbol{F}\rangle\rangle=\frac{1}{w_{0}} \int_{\partial \mathscr{B}_{0}} \boldsymbol{t}_{0} \cdot[\delta \varphi-\langle\delta \boldsymbol{F}\rangle \cdot \boldsymbol{X}] \mathrm{d} A= \\
\frac{1}{w_{0}} \int_{\partial \mathscr{B}_{0}^{\mathrm{T}}} \boldsymbol{t}_{0} \cdot[\delta \varphi-\langle\delta \boldsymbol{F}\rangle \cdot \boldsymbol{X}] \mathrm{d} A+\frac{1}{w_{0}} \int_{\partial \mathscr{B}_{0}^{\mathrm{B}}} \boldsymbol{t}_{0} \cdot[\delta \varphi-\langle\delta \boldsymbol{F}\rangle \cdot \boldsymbol{X}] \mathrm{d} A \\
+\frac{1}{w_{0}} \int_{\partial \mathscr{B}_{0}^{\mathrm{L}}} \boldsymbol{t}_{0} \cdot[\delta \varphi-\langle\delta \boldsymbol{F}\rangle \cdot \boldsymbol{X}] \mathrm{d} A+\frac{1}{w_{0}} \int_{\partial \mathscr{B}_{0}^{\mathrm{R}}} \boldsymbol{t}_{0} \cdot[\delta \varphi-\langle\delta \boldsymbol{F}\rangle \cdot \boldsymbol{X}] \mathrm{d} A=0 .
\end{gathered}
$$

This is then zero as well, because each of the first terms is zero and due to the antiperiodicity the sum of the latter two is zero. Thus the proposed hybrid boundary conditions are admissible.

\section{Computational homogenization}

The homogenization framework of the preceding section is now transferred to a computational homogenization scheme in the $\mathrm{FE}^{2}$ spirit of References [3, 9, 11, 25]. This 


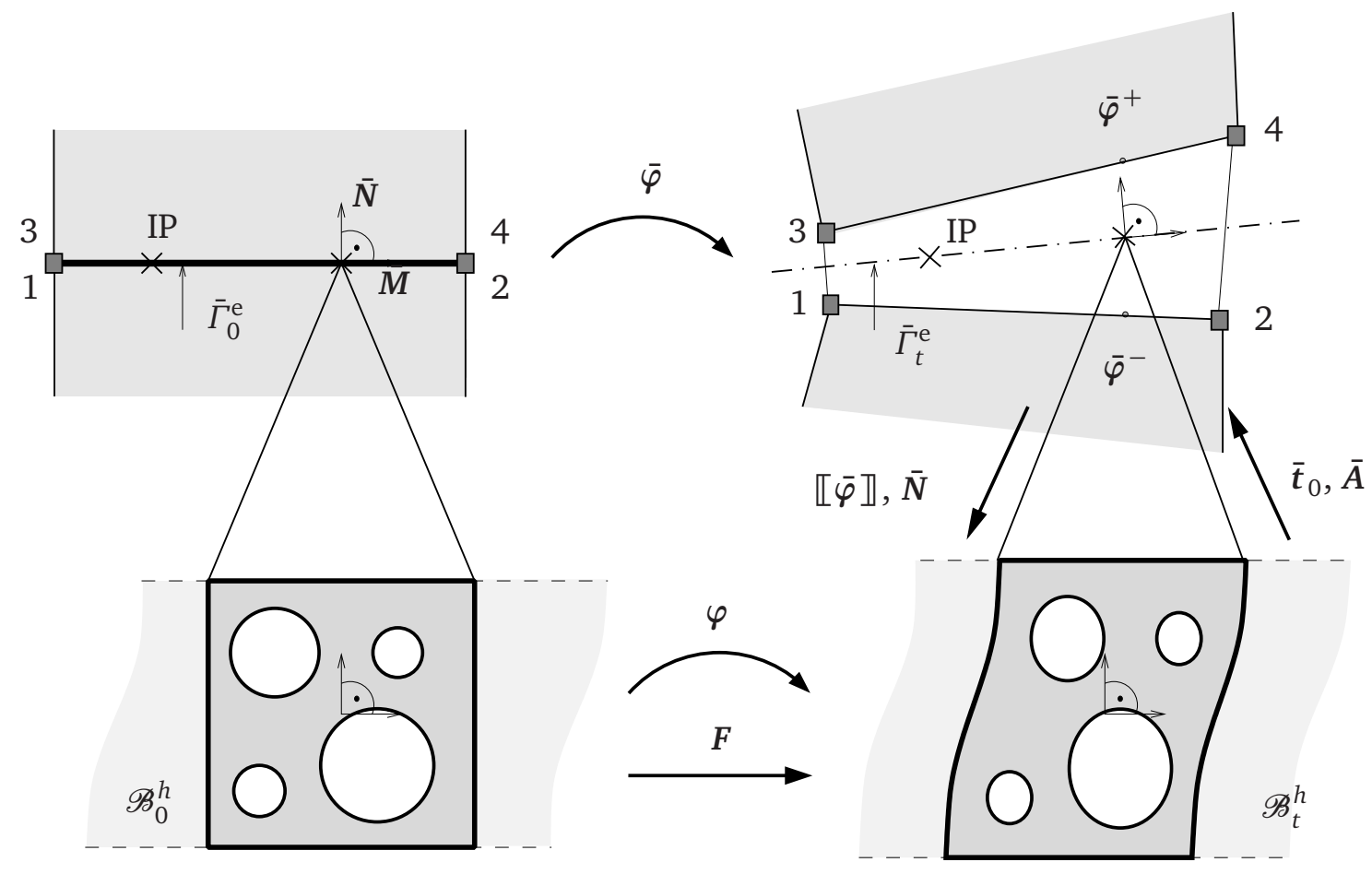

Figure 6: Computational homogenization between the interface integration point (IP) of the interface element on $\bar{\Gamma}_{0}^{\mathrm{e}}$ at the macro scale and the underlying discretized representative volume element $\mathscr{B}_{0}^{h}$ with boundary $\partial \mathscr{B}_{0}^{h}$.

consists of a nested solution scheme [11] involving both the macro- and the micro-level boundary value problems, which are solved iteratively by means of the nonlinear finiteelement method.

Situated between the bulk elements, interface elements, as they were introduced in Reference [1], represent the material layer on the macro scale. The constitutive behaviour of the bulk is assumed a priori, for which a constitutive routine is provided. Contrary, the constitutive behaviour or rather the traction-separation relation (8) of the interface element is obtained from the underlying microstructure. For this purpose, at each integration point of each interface element, the traction vector $\overline{\boldsymbol{t}}_{0}$ and the tangent operator $\bar{A}$ are evaluated by means of a computational homogenization of the underlying micro-properties in the RVE based on the macro kinematics as illustrated in Figure 6.

\subsection{Nested solution procedure}

The nested multiscale solution, which involves one macro boundary value problem and as many RVE boundary value problems as integration points in the macroscopic interface 
micro

\section{initialization}

macro BVP

- geometry and material

- set boundary conditions

- assign RVE to each interface element IP

- loop over elements

○ bulk elements

$\circ$ interface elements

$\star \quad$ loop over IP

store tangent
BVPS of RVES

- geometry, identify boundary nodes

- material(s)
$\bar{A}$

$\llbracket \bar{\varphi} \rrbracket=0$

at each RVE

- set boundary conditions

- Newton-Raphson iteration

- loop over all elements RVE assembly

- find RVE solution

- loop over prescribed nodes

- compute (macro) interface tangent

loop over load increments

- loop over load steps

$\star$ Newton-Raphson iteration

- loop over macro elements

- bulk elements

○ interface elements

* loop over IP

at each RVE

$\star$ set boundary conditions

* Newton-Raphson iteration

- loop over all elements

RVE assembly

- find solution to RVE system

* end NR iteration at convergence

$\star$ loop over prescribed nodes

store tangent

store traction

- find macro solution

* end NR iteration at convergence
$\bar{A}$

$\bar{t}_{0}$

- compute interface tangent

- compute interface traction

Figure 7: Schematic flowchart on nested multiscale solution. 
elements of Figure 6, is in particular achieved as follows.

The macro specimen $\overline{\mathscr{B}}_{0}^{h}$ is discretized with bulk finite elements in $\overline{\mathscr{B}}_{0}^{h}$, while cohesive interface elements represent the material layer on $\bar{\Gamma}_{0}^{h}$. The formulations of these interface element are well-established and for instance are described in References [23, 30, 34, 35]. Assigned to each macro integration point of each interface element, the corresponding RVE is discretized with a finite element mesh in $\mathscr{B}_{0}^{h}$. In order to handle the in-plane periodicity being part of the hybrid boundary conditions, this RVE mesh is subject to the restriction that the left and the right boundary, $\partial \mathscr{B}_{0}^{h \mathrm{~L}}$ and $\partial \mathscr{B}_{0}^{h \mathrm{R}}$, respectively, have equal arrangement. In order to achieve a geometrically nonlinear multiscale solution, at each macroscopic iteration step within a Newton-Raphson algorithm, in each integration point of each interface element the nonlinear systems of the RVEs are solved iteratively subject to the current macro deformation jump, as depicted in the schematic flow chart of Figure 7, see also Reference [8]. In particular at each integration point of each interface element on $\bar{\Gamma}_{0}^{h}$ the macro separation $\llbracket \bar{\varphi} \rrbracket$ is evaluated iteratively, being zero initially. Its increments deliver the boundary conditions to the RVE finite-element mesh, see (24). During each macro iteration step, the nonlinear micro systems are solved subject to these incremental boundary conditions. When equilibrium is obtained at the RVE level, both the homogenized macroscopic tangent operator $\bar{A}$ and the macroscopic traction vector $\overline{\boldsymbol{t}}_{0}$ of (8) at the respective integration point along the interface are computed from this solution. Precisely, the contributions of the stiffness matrix and the residual vector at the RVE boundary are extracted to this end. With the constitutive macro quantities at hand, the macro system is solved iteratively until a global solution for the current load step is obtained. In case the interface coordinate system does not coincide with the global coordinate system, the components of the separation vector in tangential and normal direction are transferred to the RVE.

\subsubsection{Nonlinear system at the macro level}

At the macro level, the global residual must vanish for all degrees of freedom $\bar{\varphi}_{I}$. Based on the weak form (7), it is obtained from an assembly of the contributions of all the bulk and all the interface elements as

$$
\overline{\mathrm{R}}_{I}=\stackrel{\bar{e}}{\mathrm{el}}_{\bar{e}=1}^{\overline{\mathscr{B}}_{\overline{\mathscr{B}}_{0}^{e}}} \int_{\boldsymbol{P}} \overline{\boldsymbol{P}} \cdot \nabla_{\bar{X}} \bar{N}_{I}^{\bar{\varphi}} \mathrm{d} V+\underset{\overline{i e}=1}{\bar{n}_{\text {iel }}} \int_{\bar{\Gamma}_{0}^{e}} \overline{\boldsymbol{t}}_{0} \cdot \bar{N}_{I}^{\llbracket \varphi \rrbracket} \mathrm{d} A-\overline{\mathbf{f}}^{\mathrm{ext}} \doteq \mathbf{0} .
$$

Herein the shape functions $\bar{N}_{I}^{\bar{\varphi}}$ act in the bulk and $\bar{N}_{I}^{\llbracket \varphi \rrbracket}$ within the interface element. The external force vector at the macro level in general contains both external traction and body forces acting on the bulk surface and volume, respectively:

$$
\overline{\mathbf{f}}_{I}^{\mathrm{ext}}=\underset{\bar{e}=1}{\overline{\mathrm{n}}_{\mathrm{el}}} \int_{\overline{\mathscr{B}}_{0}^{e}} \overline{\boldsymbol{b}}_{0} N_{I}^{\varphi} \mathrm{d} V+\int_{\partial \overline{\mathscr{B}}_{0}^{e}} \overline{\boldsymbol{t}}_{0} N_{I}^{\varphi} \mathrm{d} A .
$$


For the iterative solution of the nonlinear system of equations given by (32), we avail ourselves of a Newton-Raphson algorithm. To this end, we introduce the stiffness matrix, defined as $\overline{\mathbf{K}}_{I L}=\partial_{\bar{\varphi}_{L}} \overline{\mathbf{R}}_{I}$, and solve the linearized system of equations

$$
\overline{\mathrm{K}}_{I L} \cdot \Delta \bar{\varphi}_{L}^{h}=\overline{\mathbf{f}}_{I}^{\mathrm{ext}}-\overline{\mathbf{f}}_{I}^{\mathrm{int}} .
$$

For the given macro problem, the stiffness matrix in particular reads

$$
\overline{\mathbf{K}}_{I L}=\stackrel{\AA}{\bar{e}=1}_{\overline{\mathscr{B}}_{0}^{e}}^{\AA_{\bar{e}^{e}}} \mathrm{D}_{\overline{\boldsymbol{F}}}\left(\overline{\boldsymbol{P}} \cdot \nabla_{\bar{X}} \bar{N}_{I}^{\varphi}\right) \cdot \nabla_{\bar{X}} \bar{N}_{L}^{\bar{\varphi}} \mathrm{d} V+\mathrm{D}_{\llbracket \bar{\varphi} \rrbracket}\left(\overline{\boldsymbol{t}}_{0} \bar{N}_{I}^{\llbracket \varphi \rrbracket}\right) \bar{N}_{L}^{\llbracket \varphi \rrbracket} \mathrm{d} V .
$$

Herein the derivative $\mathrm{D}_{\bar{F}}\left(\overline{\boldsymbol{P}} \cdot \nabla_{\bar{X}} \bar{N}_{I}^{\varphi}\right)$ for the bulk can be evaluated directly based on an a priori constitutive assumption. Contrary, the material tangent operator $\mathrm{D}_{\llbracket \bar{\varphi} \rrbracket}\left(\overline{\boldsymbol{t}}_{0}\right)$ of each interface element calls to be determined from the underlying RVE in a computational homogenization at each integration point. In particular, a Gauss quadrature is used for the numerical integration within the interface elements, whereas other numerical integration schemes [30] go beyond the scope of the current contribution. Within the loop over the Gauss points, instead of a material routine, the underlying RVE programme is called in order to retrieve the tangent operator as well as the traction vector. To this end, the current trial value of the macro separation are passed to this RVE routine and the resulting procedure is described in the following section.

\subsubsection{Solution of the nonlinear RVE problem}

For each macroscopic interface integration point the material response needs to be evaluated on the underlying RVE. To this end, each RVE receives the current trial separation vector which is translated to a boundary condition according to Equation (24). Only after the RVE system is solved subject to this boundary condition, the sought-for macro material information can be extracted.

In the system subject to the Dirichlet boundary conditions stemming from the macro level, the finite-element stiffness matrix and the residual vector of the RVE problem are assembled from the individual finite-element contributions in a standard manner:

$$
\begin{aligned}
\mathrm{R}_{I} & =\underset{e=1}{\stackrel{n_{\mathrm{el}}}{A}} \int_{\mathscr{B}_{0}^{\mathrm{e}}} \boldsymbol{P} \cdot \nabla_{X} N_{I} \mathrm{~d} V-\mathbf{f}_{I}^{\mathrm{ext}} \doteq \mathbf{0}, \\
\mathrm{K}_{I L} & =\underset{e=1}{\stackrel{n_{\mathrm{el}}}{\boldsymbol{A}}} \int_{\mathscr{B}_{0}^{\mathrm{e}}} \mathrm{D}_{\boldsymbol{F}}\left(\boldsymbol{P} \cdot \nabla_{X} N_{I}^{\varphi}\right) \cdot \nabla_{X} N_{L}^{\varphi} \mathrm{d} V,
\end{aligned}
$$

with $N_{I}$ and $N_{L}$ being the shape functions for the trial and test function respectively. Thereby a priori constitutive formulations are used to compute the stress $\boldsymbol{P}$ and the material operator $\mathrm{D}_{F} \boldsymbol{P}$ in each element. 
In order to account for the periodicity (25), during the iterative solution the entire system of equations is transformed into a reduced system of independent degrees of freedom exclusively:

$$
\mathrm{K}_{I L}^{\star} \cdot \Delta \varphi_{L}^{\star}=\mathrm{R}^{\star} .
$$

with $\varphi^{\star}=\varphi_{\mathrm{i}}$ comprising the independent degrees of freedom only. As proposed by Kouznetsova et al. $[8,10]$ this is accomplished by means of a dependency matrix, which relates the dependent with the independent nodal displacements as

$$
u_{\mathrm{d}}=\mathrm{D}_{\mathrm{di}} \cdot \boldsymbol{u}_{\mathrm{i}} .
$$

With the deformation map $\varphi$ being the actual degree of freedom, we have made use of the fact, that the Newton-Raphson algorithm deals with increments and thereby $\Delta \boldsymbol{u}=$ $\Delta \varphi$. In the transformed system (38), the reduced stiffness matrix and residual vector are computed as

$$
\begin{aligned}
& \mathrm{K}^{\star}=\mathrm{K}_{\mathrm{ii}}+\mathrm{D}_{\mathrm{di}}^{\mathrm{t}} \cdot \mathrm{K}_{\mathrm{id}}+\mathrm{K}_{\mathrm{id}} \cdot \mathrm{D}_{\mathrm{di}}+\mathrm{D}_{\mathrm{di}}^{\mathrm{t}} \cdot \mathrm{K}_{\mathrm{dd}} \cdot \mathrm{D}_{\mathrm{di}} \\
& \mathrm{R}^{\star}=\mathrm{R}_{\mathrm{i}}+\mathrm{D}_{\mathrm{di}} \cdot \mathrm{R}_{\mathrm{d}}
\end{aligned}
$$

For the material layer RVE under the boundary conditions proposed in Section 4.3.1, the independent degrees of freedom comprise the degrees of freedom of all boundary nodes at the top and bottom (including all corner nodes), on the left, as well as all interior nodes. Complementarily, the right boundary nodes supply the set of dependent degrees of freedom, as illustrated in Figure 8.

$$
I_{\mathrm{i}} \in\{\text { top, left, bottom, interior }\}, \quad I_{\mathrm{d}} \in\{\text { right }\}
$$

In this reduced system, the displacement-boundary conditions (24) stemming from the interface deformation, are imposed at the top and bottom nodes in order to find a solution. The vector of unknowns is updated, before the independent and the dependent degrees of freedom are gathered. In this way the nonlinear micro system of equations is iteratively solved until equilibrium is reached. The procedure is summarized in Table 1.

Remark 5.1 Other techniques to enforce the periodicity have been proposed in the literature. For instance Miehe [21] uses Lagrange multipliers. Another alternative lies in the modification of the basis functions of the respective degrees of freedom on the positive and negative edge of the RVE, see Reference [33].

\subsection{Homogenized macro quantities}

With the respective solved RVE system at hand, we obtain the sought-for macroscopic quantities, i.e. the traction vector (6) 2 and the tangent operator (8) at the superordinate interface integration point from a computational homogenization. Therefore the prescribed nodes at the top and bottom of the RVE (pn) and the free nodes (fn) given by all other independent nodes are identified,

$$
I_{\mathrm{pn}} \in\{\text { top, bottom }\}, \quad I_{\mathrm{fn}} \in\{\text { left, interior }\},
$$



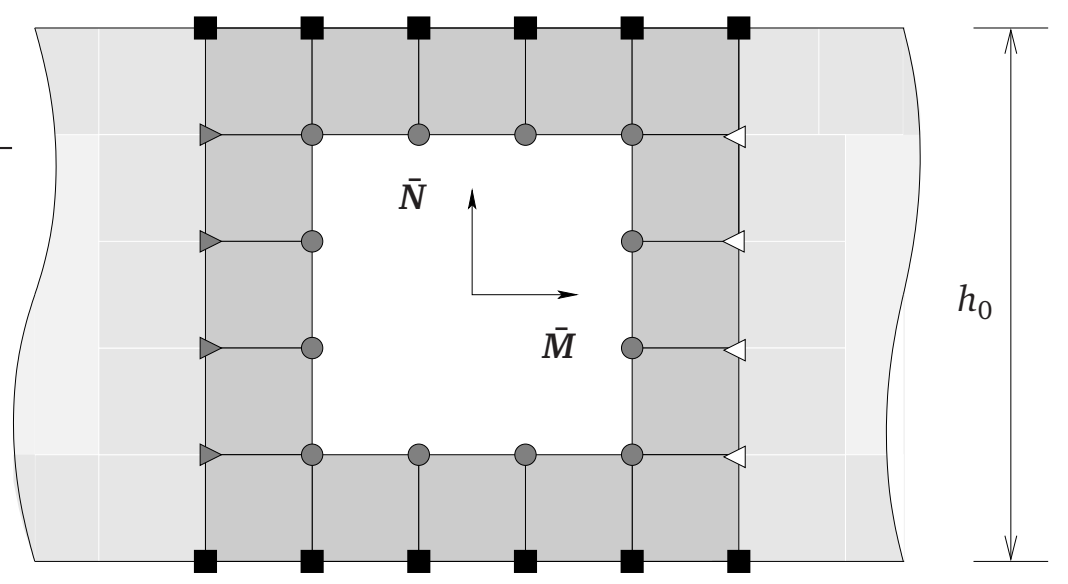

independent dofs:

- top, bottom

$\triangleright$ left-side

- interior

dependent dofs:

$\triangleleft$ right-side

Figure 8: Simple RVE mesh displaying the independent degrees of freedom, which comprise the prescribed nodes on the top and the bottom boundary (black-filled), the left-hand side nodes as well as the interior nodes, and the dependent degrees of freedom (white-filled), which only consist of the right-hand side nodes of the RVE.

Table 1: Flow chart on the numerical treatment of the periodicity. $\varphi^{(0)}$ is the vector with the deformation dofs before and $\varphi^{(1)}$ after the solution of the current step.

0 . initialization: get dofs $\varphi^{(0)}$ from coordinates of last step (initially $\varphi^{(0)}=X$ )

1. get $\mathrm{K}^{\mathrm{e}}$ and $\mathrm{R}^{\mathrm{e}}$ from individual RVE elements

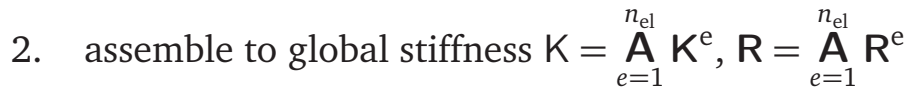

3. separate $\mathbf{K}$ and $\mathbf{R}$ into independent and dependent dofs

$$
K=\left[\begin{array}{ll}
K_{i i} & K_{i d} \\
K_{d i} & K_{d d}
\end{array}\right], \quad R=\left[\begin{array}{l}
R_{i} \\
R_{d}
\end{array}\right]
$$

4. get stiffness matrix $\mathrm{K}^{\star}$ for independent dofs by transformation

get residual vector $\mathbf{R}^{\star}$ for the transformed system

extract all independent dofs $\varphi_{i}^{(0)}$

5. solve system of independent dofs and obtain $\varphi_{\mathrm{i}}^{(1)}$

6. update dependent dofs : $\varphi_{\mathrm{d}}^{(1)}=\mathrm{D}_{\mathrm{di}} \cdot\left[\varphi_{\mathrm{i}}^{(1)}-X_{\mathrm{i}}\right]+X_{\mathrm{d}}$

7. gather all dofs $\varphi^{(1)}=\left[\varphi_{\mathrm{i}}^{(1)}, \varphi_{\mathrm{d}}^{(1)}\right]^{\mathrm{t}}$

8 check convergence

$\triangleright$ if residual norm of system in independent dofs $>$ TOL set $\varphi^{(0)}=\varphi^{(1)}$, go to step 1 and repeat procedure

$\triangleright$ else if residual norm of system in independent dofs $\leq$ TOL

RVE system is solved 
in the system, as it was solved for the independent degrees of freedom:

$$
\left[\begin{array}{cc}
\mathrm{K}_{\mathrm{pn}, \mathrm{pn}}^{\star} & \mathrm{K}_{\mathrm{pn}, \mathrm{fn}}^{\star} \\
\mathrm{K}_{\mathrm{fn}, \mathrm{pn}}^{\star} & \mathrm{K}_{\mathrm{fn}, \mathrm{fn}}^{\star}
\end{array}\right] \cdot\left[\begin{array}{c}
\Delta \varphi_{\mathrm{pn}}^{\star} \\
\Delta \varphi_{\mathrm{fn}}^{\star}
\end{array}\right]=\left[\begin{array}{c}
\Delta f_{\mathrm{pn}}^{\star} \\
0
\end{array}\right]
$$

Note that at the solved state, the internal nodal forces at the prescribed nodes represent the reaction forces. With this the system can be further condensed into the contribution of the prescribed nodes only

$$
\mathrm{K}^{\diamond} \cdot \Delta \varphi_{\mathrm{pn}}=\Delta \mathbf{f}^{\diamond} .
$$

Therein stiffness matrix $\mathbf{K}^{\diamond}$ and the external nodal force vector $\mathbf{f}^{\diamond}$ are determined as

$$
\mathrm{K}^{\diamond}=\mathrm{K}_{\mathrm{pn}, \mathrm{pn}}^{\star}-\mathrm{K}_{\mathrm{pn}, \mathrm{fn}}^{\star} \cdot\left(\mathrm{K}_{\mathrm{fn}, \mathrm{fn}}^{\star}\right)^{-1} \cdot \mathrm{K}_{\mathrm{fn}, \mathrm{pn}}^{\star}, \quad \quad \mathbf{f}^{\diamond}:=\mathrm{f}_{\mathrm{pn}}^{\star} .
$$

This allows to obtain the resulting traction from the reaction forces at these prescribed nodes and the tangent being the operator that, applied on the macro separation increment, yields the the resulting traction increment.

\subsubsection{Traction}

With the reaction force at the prescribed boundary nodes (46) at hand, we retrieve the homogenized macro traction vector (21) by means of the average of the Piola stress (14) as

$$
\overline{\boldsymbol{t}}_{0}=\frac{1}{V_{0}} \sum_{I_{\mathrm{pn}}}^{n_{\mathrm{pn}}}\left[\mathrm{f}_{I_{\mathrm{pn}}}^{\diamond} \otimes X_{I_{\mathrm{pn}}}\right] \cdot \overline{\boldsymbol{N}}
$$

Thereby the summation runs over all $n_{\mathrm{pn}}$ prescribed nodes on the top and bottom boundaries of the representative volume element, $\partial \mathscr{B}_{0}^{h^{\mathrm{T}}} \cup \partial \mathscr{B}_{0}^{h^{\mathrm{B}}}$.

\subsubsection{Tangent}

For the RVE we are seeking the tangent operator in the incremental formulation of (8) for a finite increment $\Delta \overline{\boldsymbol{t}}_{0}$ as it is used in a Newton-Raphson scheme. From the increment of the macro traction (47) with the reduced system (45) we directly obtain the tangent as

$$
\bar{A}=\frac{1}{w_{0} h_{0}^{2}}[\bar{N} \otimes \bar{N}]:\left[\sum_{I_{\mathrm{pn}}}^{n_{\mathrm{pn}}} \sum_{K_{\mathrm{pn}}}^{n_{\mathrm{pn}}}\left[X_{K_{\mathrm{pn}}} \otimes X_{I_{\mathrm{pn}}}\right] \otimes \mathrm{K}_{I_{\mathrm{pn}} K_{\mathrm{pn}}}^{\diamond}\right] .
$$

Therein the prescribed deformation boundary conditions (24) at nodes $K_{\mathrm{pn}}$ were considered. Hereby the summation runs over all nodes $I_{\mathrm{pn}}, K_{\mathrm{pn}}$ on the prescribed top and bottom boundary $\partial \mathscr{B}_{0}^{h^{\mathrm{T}}} \cup \partial \mathscr{B}_{0}^{h^{\mathrm{B}}}$. For further details on the set of equations constituting the computational framework, the reader is referred to Reference [6]. 


\section{Numerical examples}

In order to illustrate the proposed computational homogenization procedure, numerical examples are presented. Underlying to a material layer, sample microstructures with either voids or inclusions are simulated. First, we study the proper choice of the RVE at the example of a tailored microstructure within a periodic material layer, Section 6.1, with one interface element, as illustrated in Figure 9. More complex macro boundary value problems are studied in Sections 6.2 and 6.3. At the macro level, the cohesive interface is embedded in a bulk finite-element mesh with linear, respectively bi-linear approximations. The underlying microstructure or rather the representative volume elements are discretized by bi-quadratic bulk elements.

\subsection{Choice of the RVE}

Within the simplified multiscale framework that is shown in Figure 9, a material layer with a periodic microstructure is considered. In the darker grey region of the periodic microstructure in Figure 10, the Young's modulus is chosen five times as high as in the lighter one. For this periodic microstructure, the proper choice of the RVE is investigated. To this end, out of the various possible options, one non-symmetric and one symmetric RVE are chosen, which both possess a width to height ratio of $2 / 3$. With the aid of the simplest possible macro problem, a single interface element, which is shown in Figure 9, we evaluate the homogenized macro response to fully prescribed mixed-mode loading, $\bar{u}_{\bar{M}}=5 \bar{u}_{\bar{N}}$. This deformation is applied step-wise until a final shearing of $\bar{u}_{\bar{M}}=0.4 h_{0}$ is reached. The response is evaluated for both cases, the non-symmetric and the symmetric RVEs. Figure 11 shows the spatial meshes of these after the first load step of $\bar{u}_{\bar{M}}=0.04 h_{0}$.

The stress in the RVE is considered first. Figure 12 shows the components of the

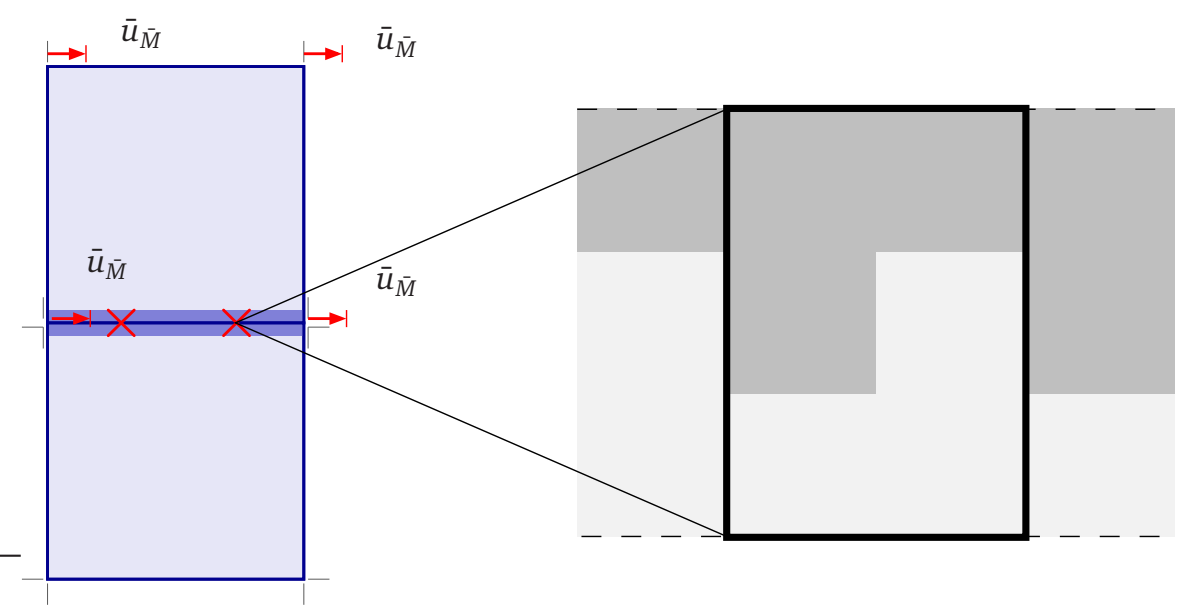

Figure 9: Benchmark problem for multiscale framework: Single interface element subjected to shear with a arbitrary sample RVE underlying to each of its integration points. 


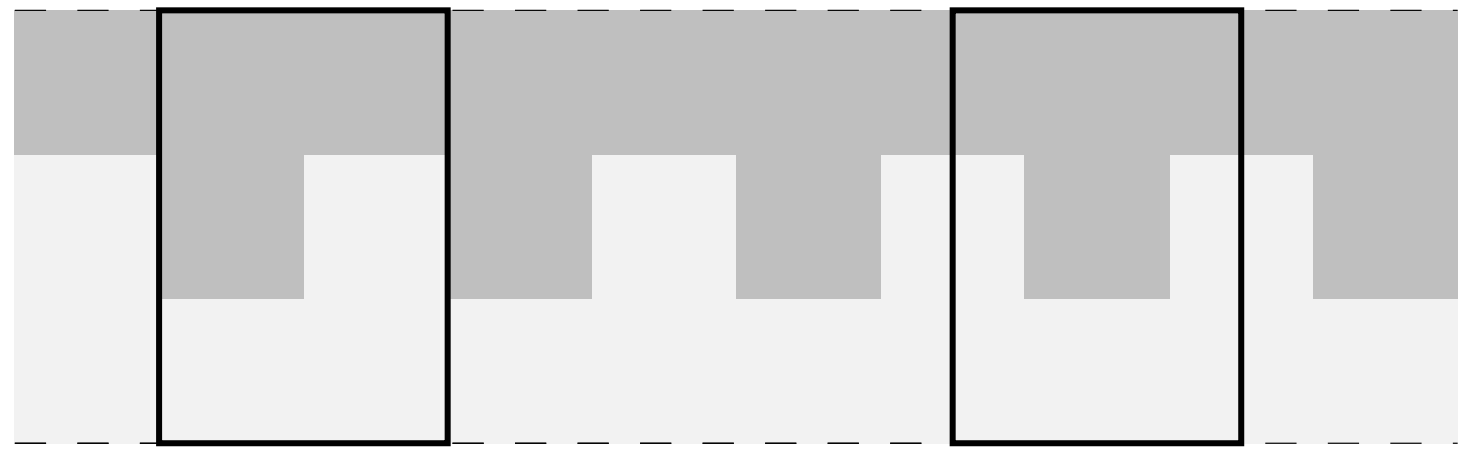

Figure 10: Periodic microstructure: choice of non-symmetric RVE vs. symmetric RVE.

(a)

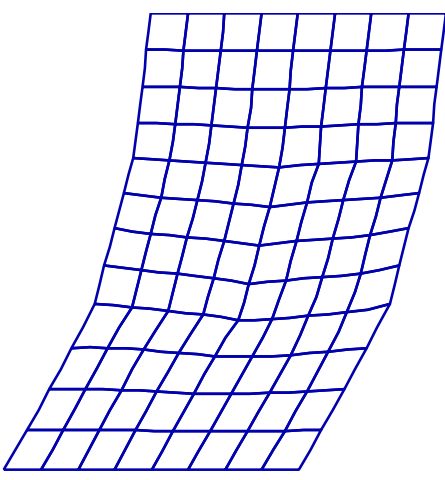

(b)

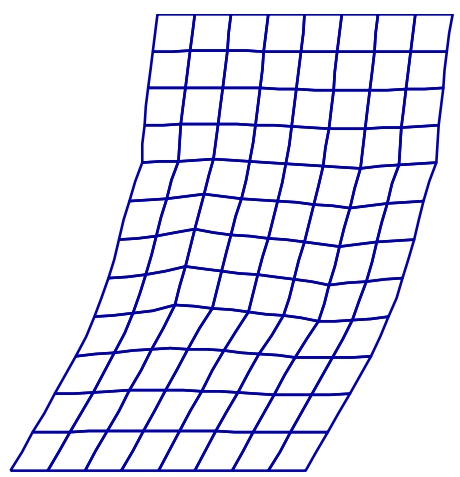

Figure 11: Spatial meshes of (a) non-symmetric RVE vs. (b) symmetric RVE at $\bar{u}_{\bar{M}}=$ $5 \bar{u}_{\bar{N}}=0.04 h_{0}$.

Cauchy type stress in the RVE. In the first row the stress in the non-symmetric and in the second row that of the symmetric RVE are plotted. Repetitive features in the stress distribution can be recognized. However, especially close to the lateral boundaries of the respective RVE, the stress patterns are not fully congruent. This is attributed to the stress interpolation algorithm in the plot routine, which does not account for the periodicity along the lateral edges.

Although the stress distributions do not appear entirely identical, the macro response is. This is shown with the resulting traction-separation laws from the computational homogenization (47). It is compared for both RVE choices in Figure 13. The response in normal direction obeys a nonlinear relation, whereas the traction-separation curve for the shear is approximately linear. Both the tangential and the normal tractionseparation curves of the two RVEs prove to coincide. Consequently, despite the slightly deviating stress, this choice of the RVE in the infinite layer has no impact on the macro response. 
(a)

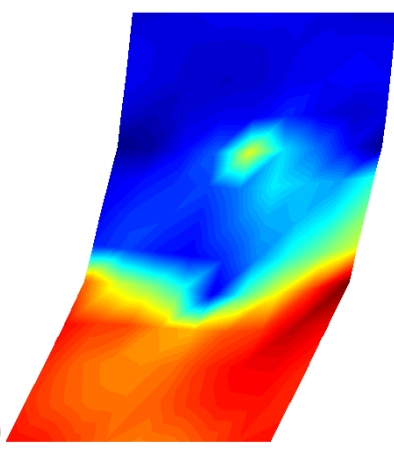

$\sigma_{\bar{M} \bar{M}}$

(b)

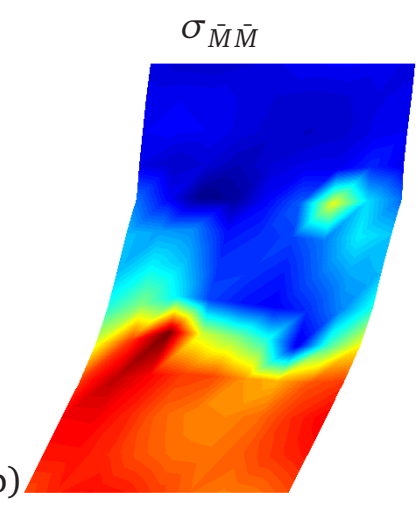

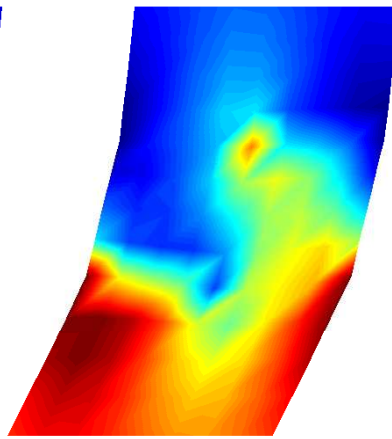

$\sigma_{\bar{N} \bar{N}}$

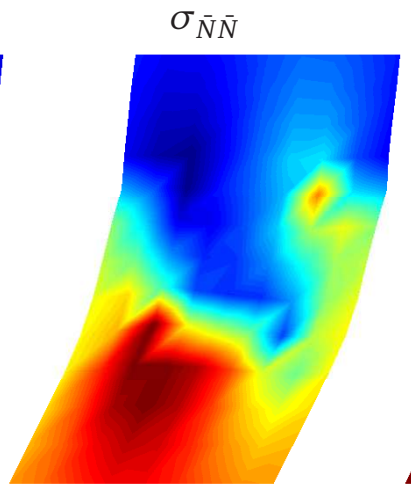

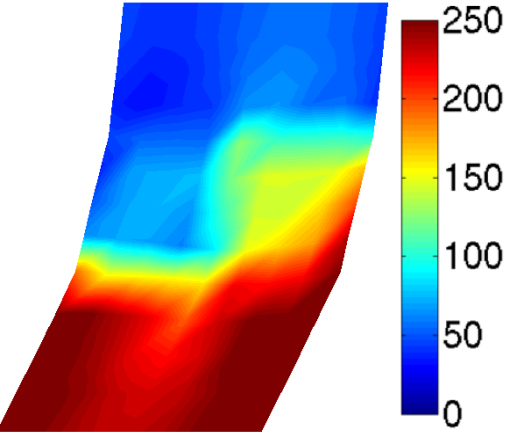

$\sigma_{\bar{M} \bar{N}}=\sigma_{\bar{N} \bar{M}}$

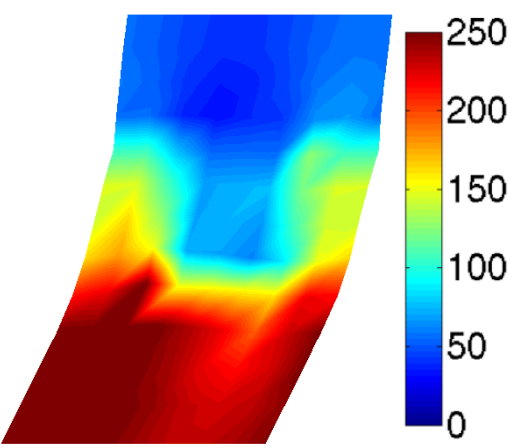

Figure 12: Cauchy stress components for (a) non-symmetric and (b) symmetric RVE.

(a,

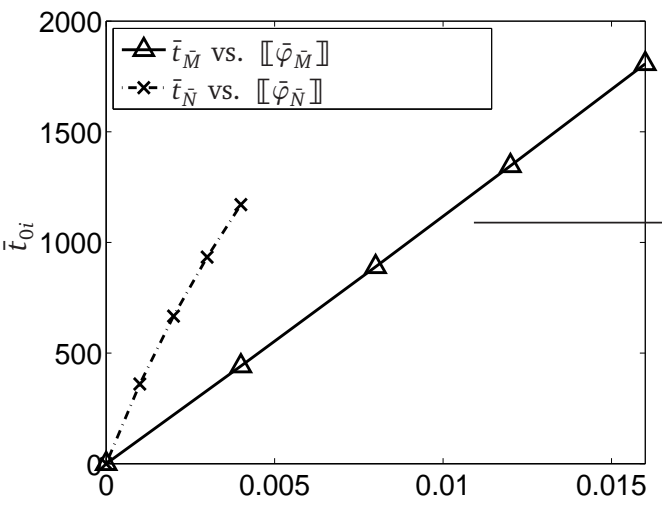

$\llbracket \bar{\varphi}_{i} \rrbracket$

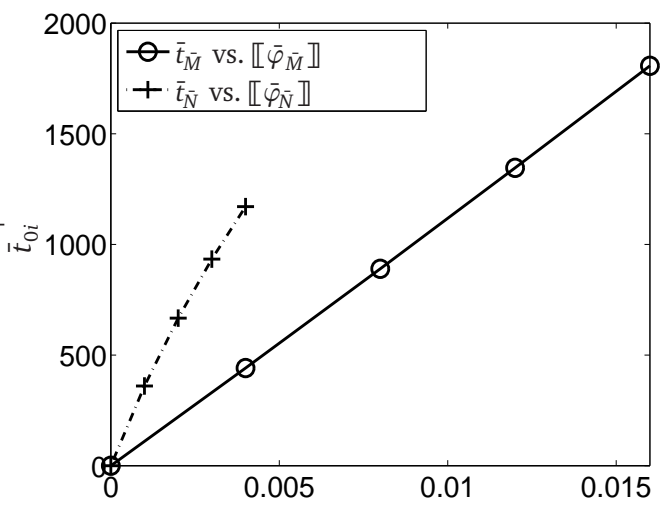

(b,

$\llbracket \bar{\varphi}_{i} \rrbracket$

Figure 13: Traction-separation curve with (a) non-symmetric and (b) symmetric RVE. 


\subsection{Infinite material layer under shear-dominated mixed-mode loading}

In order to simulate a straight material layer of large in-plane extension, a single column of elements, comprising one interface element, is modelled periodically on the macro level, as depicted in Figure 14. The total height of the shear layer is given as $\bar{H}_{0}=20 h_{0}$. For opposite nodes at the left and right side, periodic deformations are enforced. In this way, the column represents a small portion of a layer with ideally infinite in-plane extension. The deformation of the problem is prescribed at the top and the bottom $\left(\overline{\boldsymbol{u}}^{\text {pre,T}}=-\overline{\boldsymbol{u}}^{\text {pre,B }}\right)$, with a shear-dominated mixed mode, with the horizontal or rather tangential displacement being ten times the tensile or rather normal displacement. This deformation is applied step-wise, until a final tangential deformation of $\bar{u}_{\bar{M}}^{\mathrm{pre}, \mathrm{T}}=0.2 \bar{w}_{0}$ is reached.

Different micro meshes are examined: First, microstructures with a void of different shape and size are simulated and compared to the response of a homogeneous microstructure. Thereafter, the shear layer is investigated with RVEs containing inclusions of higher stiffness and different distributions. At the macro level, bi-linear shape functions are used.

\subsubsection{Microstructures with voids}

The problem is first studied for microstructures with voids. A homogeneous RVE of width $w_{0}=0.1 h_{0}$ is compared with two square RVEs with each containing a centred circular hole of $5 \%$ and $25 \%$ void ratio, respectively, and another square specimen with a centred lentil-shaped void. These microstructures are discretized with bi-linear finite elements. The macro material parameters are chosen to be $\bar{E}=100 E, \bar{v}=v=0.3$.

For a macro displacement load of $\overline{\boldsymbol{u}}^{\text {pre,T }}=-\overline{\boldsymbol{u}}^{\text {pre, B }}=0.2 \bar{w}_{0} \overline{\boldsymbol{M}}+0.02 \bar{w}_{0} \overline{\boldsymbol{N}}$, in Figure 15(b)-(d) the respective spatial macro meshes are plotted, whereas the corresponding spatial micro meshes are shown in Figure 16. Based on the different size and shape of the voids, the stiffness of the specimen differs. This is qualitatively reflected in the deformed meshes. The weaker the RVE reacts, the stronger is the deformation localized in the material layer. The differently stiff response is quantitatively analyzed in Figure 17. The force- displacement curves in tangential and normal direction are evaluated for the top nodes of the macro specimen. The specimen with the lens-shaped void yields the least stiff response, whereas for decreasing size of the void, the stiffness increases.

\subsubsection{Microstructures with inclusions}

Next, underlying to the material layer situated within the periodic macro shear layer, microstructures equipped with inclusions are examined. These possess a greater stiffness than the surrounding matrix material in the material layer. The different material meshes are schematized in Figure 18. In the darker elements, Young's modulus is chosen five times that of the light-grey elements, $E_{2}=5 E_{1}=\bar{E} / 200$, while Poisson's ratio is chosen equally as $\bar{v}=v_{1}=v_{2}=0.3$. 


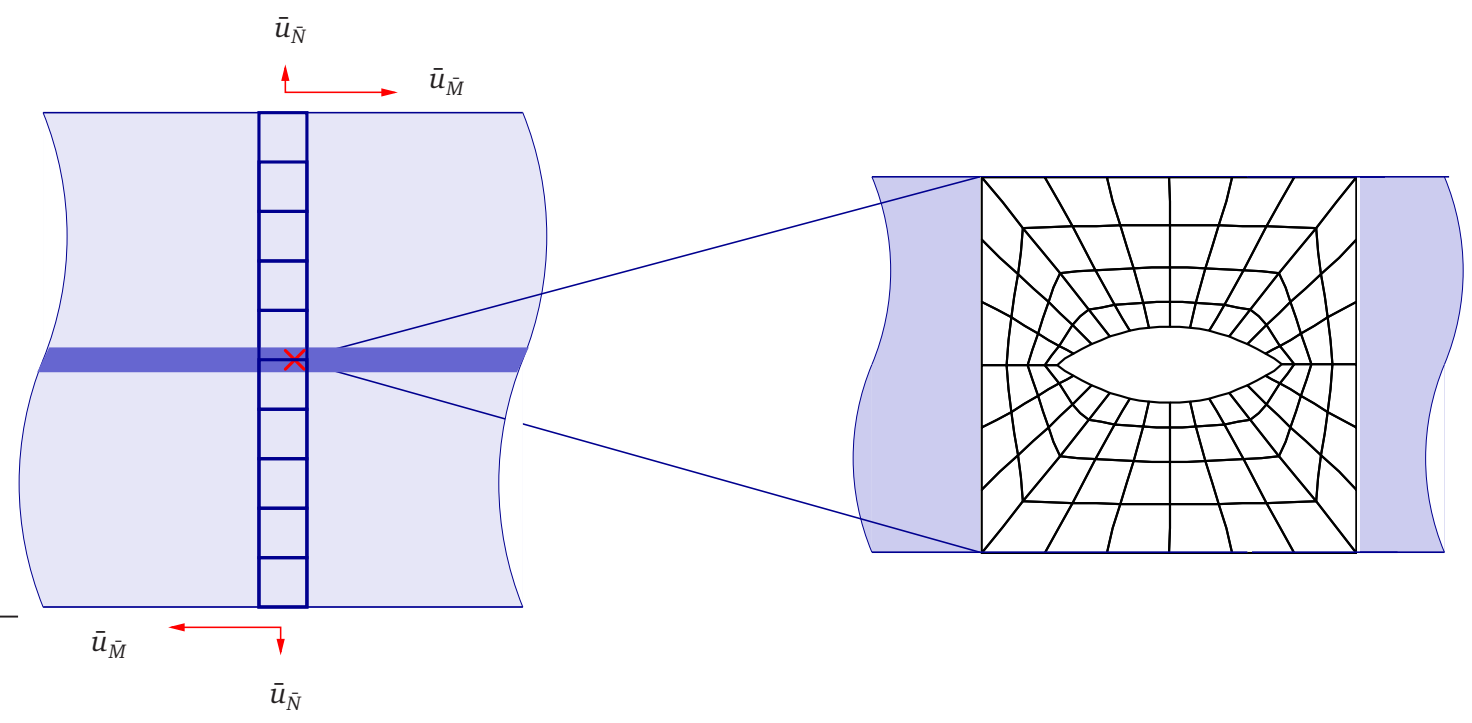

Figure 14: Infinite periodic shear layer including material layer: multiscale boundary value problem.

As for the microstructures with voids in Section 6.2.1, the force-displacement curves at the top of the macro specimen are compared here in Figure 19. Depending on the size and distribution of the inclusions, the resulting macroscopic force-displacement curves differ from each other. As expected, the specimen with the largest inclusion ratio, Figure 18(b), exhibits the stiffest behaviour. 
(a)

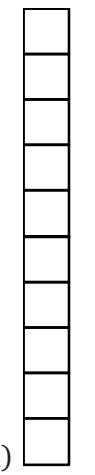

(b)

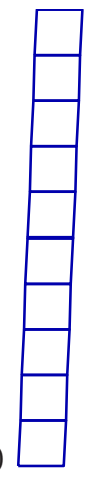

(c)

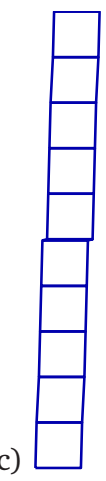

(d)

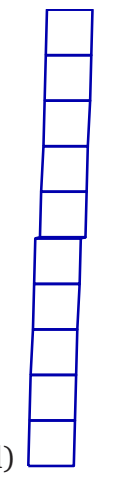

(e)

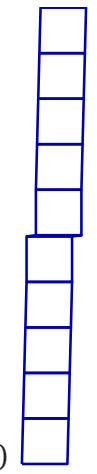

Figure 15: Shear layer with interface: (a) material macro mesh; spatial macro mesh at $\bar{u}_{\bar{M}}=0.2 \bar{w}_{0}$ for different microstructures: (b) benchmark homogeneous microstructure, (c) 5\% void, (d) 25\% void, (e) lens-shaped void.
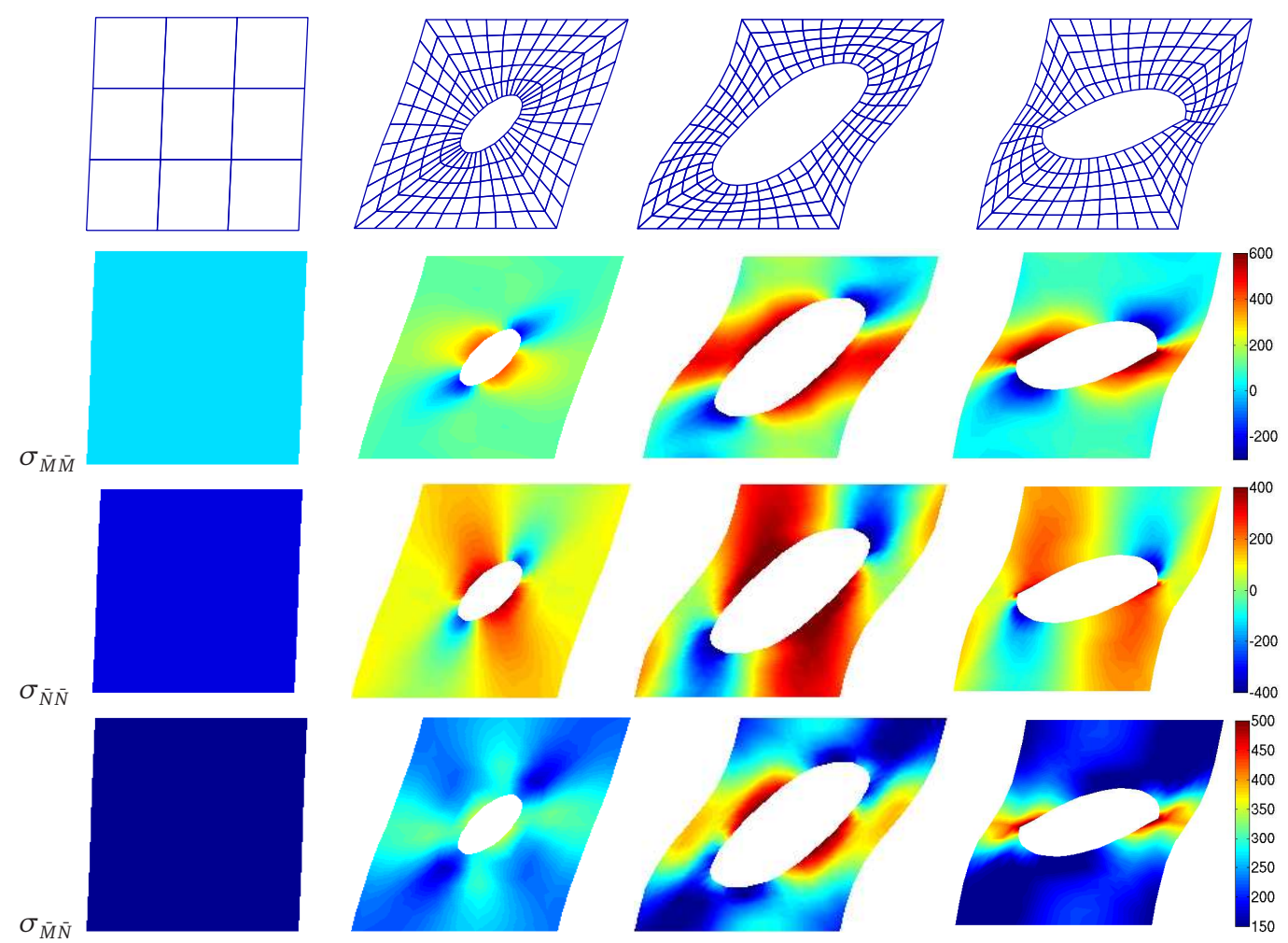

Figure 16: Shear layer with interface: spatial RVEs and Cauchy-stress $\sigma$. 
(a)

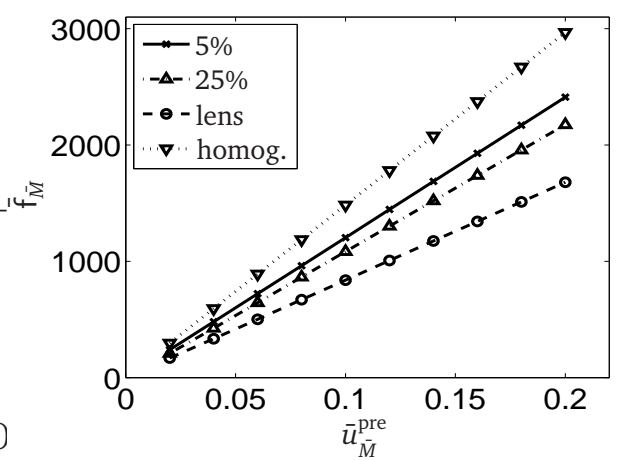

(b)

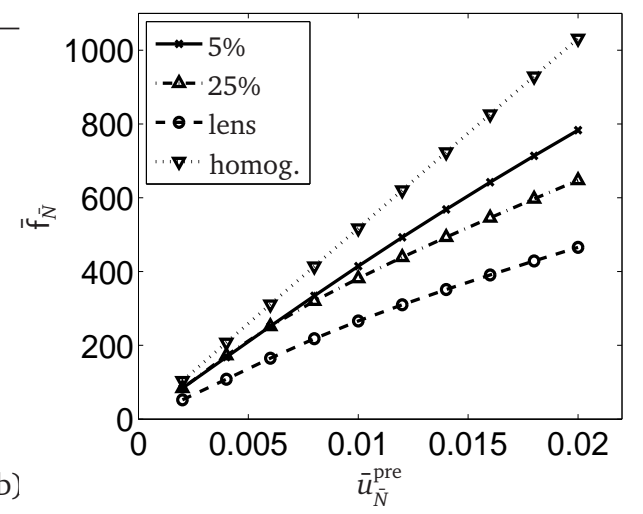

Figure 17: Shear layer with interface, underlying RVE with voids: force-displacement curves at top node, (a) tangential, (b) normal component

(a)

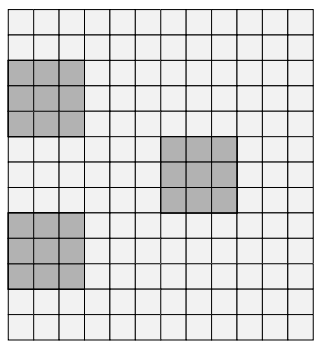

(d)

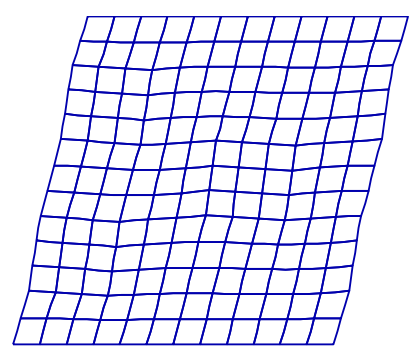

(b)

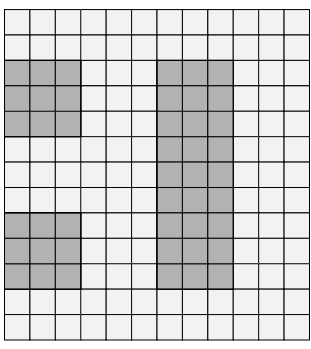

(e)

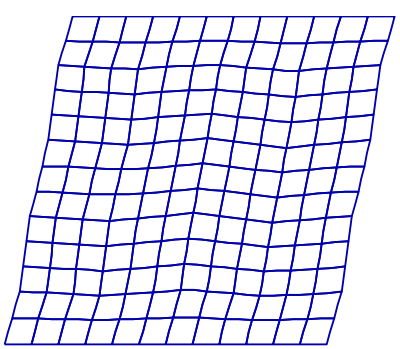

(c)

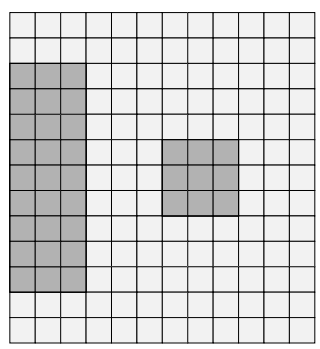

(f)

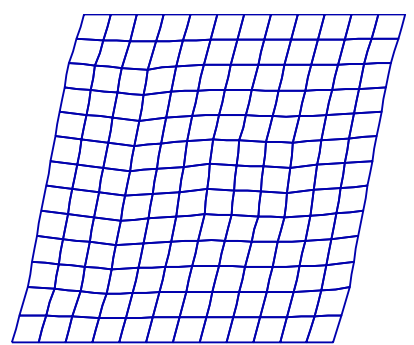

Figure 18: RVEs for microstructure with inclusions: (a)-(c) material meshes with heterogeneous material properties, (d)-(e) corresponding spatial meshes. 
(a)

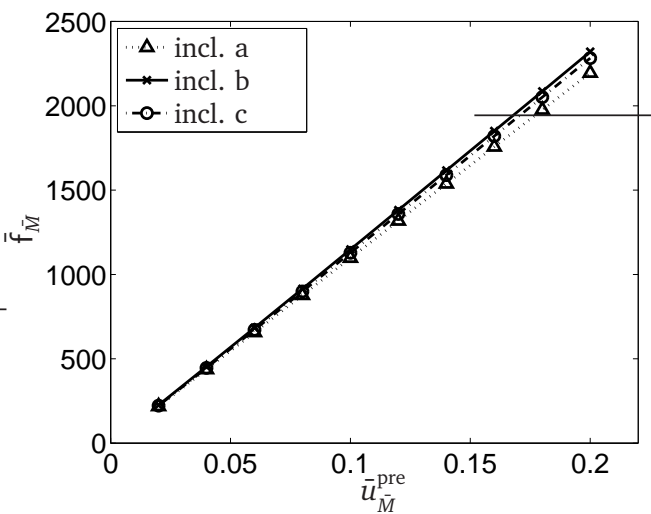

(b)

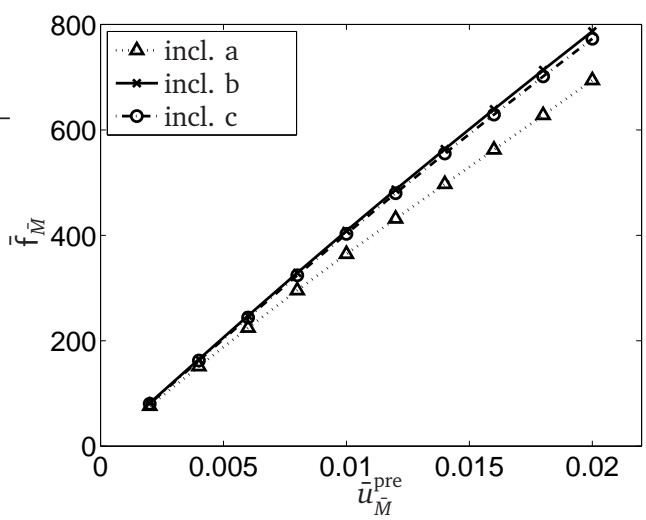

Figure 19: Macro force-displacement curves, (a) tangential and (b) normal components. 


\subsection{Macrostructure with material layer next to a hole}

As the final example, we consider a square specimen with an initially circular centred hole at the macro level with a material layer located at the lateral sides of this hole, shown in Figure 20. The response of this layer is evaluated based on its underlying microstructure, which is represented by a square RVE with a centred lens shaped void. As also illustrated in Figure 20, we consider two different orientation of this RVE: a horizontal (or rather tangential) and a vertical one. In the discretized macro specimen, three interface elements are located at each side of the hole. At each of their integration points, the respective RVE is used to evaluate the material response of the material layer. In contrast to the similar RVE of Section 6.2.1, Figure 16, here fewer elements with biquadratic shape functions are chosen. The total height of the macro specimen is chosen to be $\bar{H}_{0}=10 h_{0}$, with $h_{0}$ being the height of the material layer. For the choice of a square RVE, its width then results as $w_{0}=h_{0}=\bar{H}_{0} / 10$.

In order to identify the respective spatial RVEs assigned to the interface element integration points along the interface element, we introduce the coordinate $\bar{\Xi}=2 \bar{X}_{\bar{M}} / \bar{w}_{0}$ that denotes the relative initial location compared to half the width of the macro specimen as illustrated in Figure 20. Figure (21) displays the corresponding spatial RVE meshes at the integration point coordinates. Knowing that the model prevents a lateral deformation of the RVE, we observe that the RVE with the horizontally oriented void undergoes larger deformations in loading direction. This qualitative result is supported by the quantitative curves in in Figure 22. Here, besides the spatial macro mesh, the corresponding homogenized tractions $\bar{t}_{\bar{N}}$ and the separations $\llbracket \bar{\varphi}_{\bar{N}} \rrbracket$ at these macro Gauss points are plotted versus their position $\bar{\Xi}$. As expected, closer to the macroscopic hole, both the traction and the deformation increase more steeply. Furthermore, the orientation of the lens-shaped void plays a significant role. The horizontally oriented void attracts more separation and less traction, while the slope of the traction-separation curve is less steep. Consequently, this specimen involves a weaker response than that with the vertically oriented void. This is also reflected in the two resulting tractionseparation curves at the macro integration point closest to the hole, $\bar{t}_{\bar{N}}$ versus $\llbracket \bar{\varphi}_{\bar{N}} \rrbracket$. It can be observed that by their orientation, heterogeneities in the microstructure can yield anisotropic effects in the global response of the material layer. 


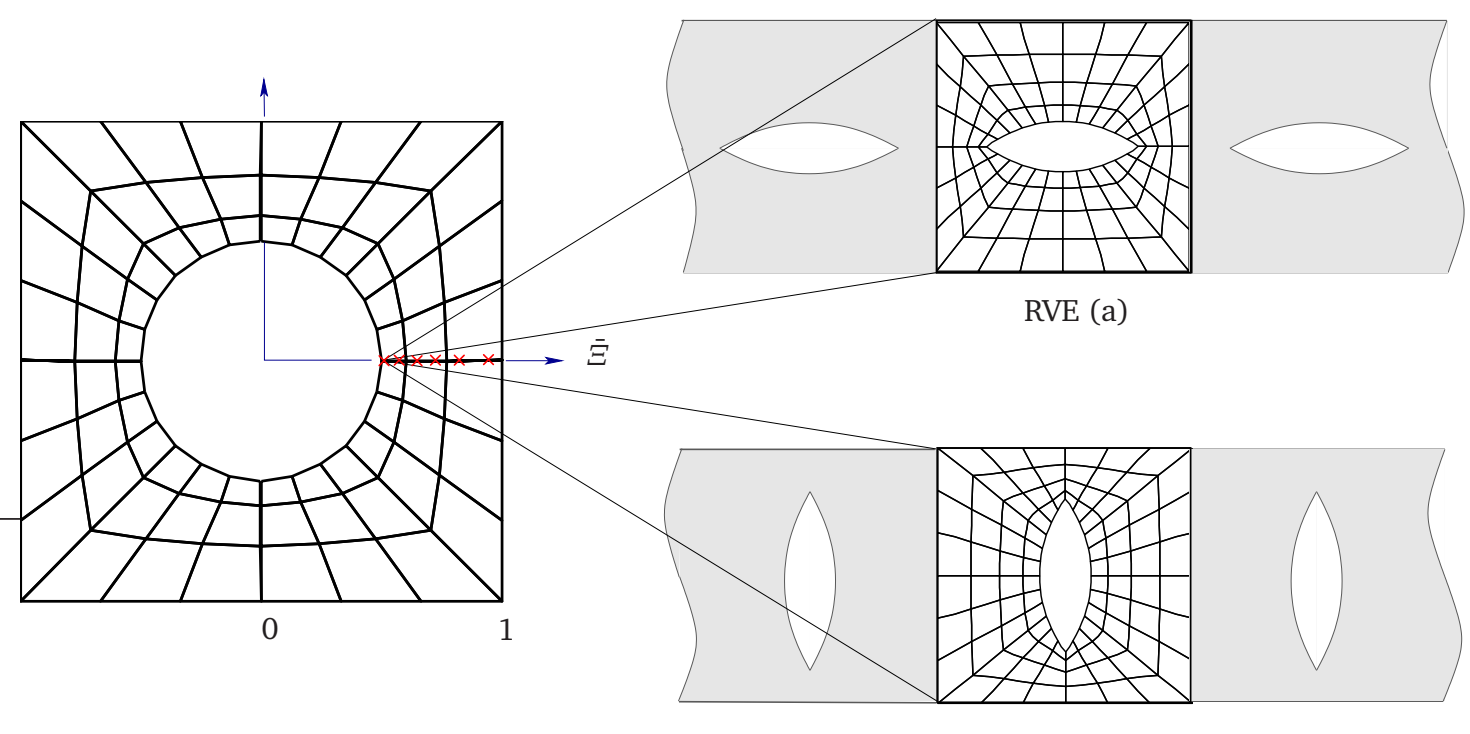

RVE (b)

Figure 20: Multiscale boundary value problem with the two RVEs under investigation, material finite-element mesh: Macro specimen with circular void and horizontal interface layer, RVE (a) with horizontally and RVE (b) with vertically oriented lens-shaped void. 
(a)

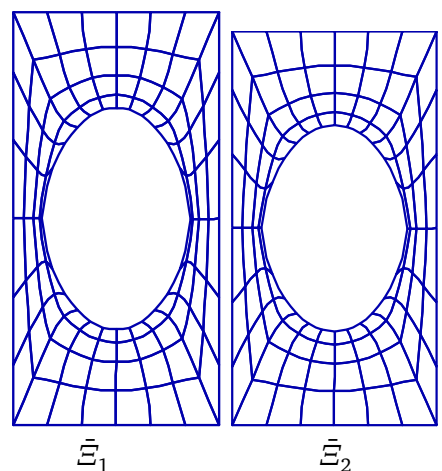

(b)

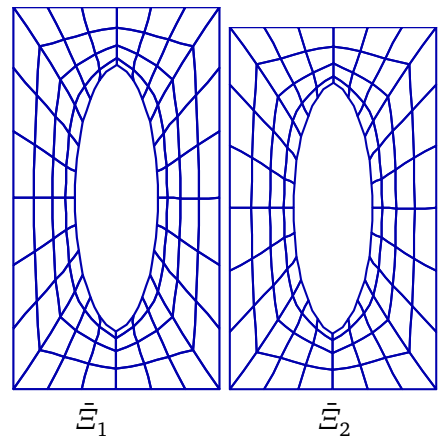

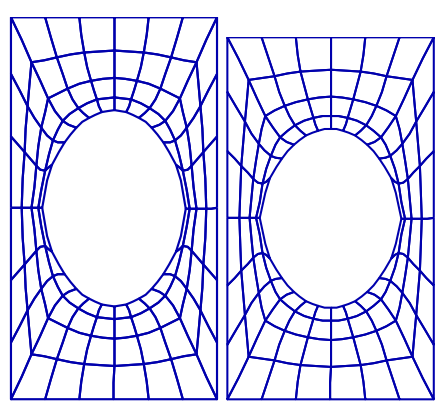

$\bar{\Xi}_{4}$

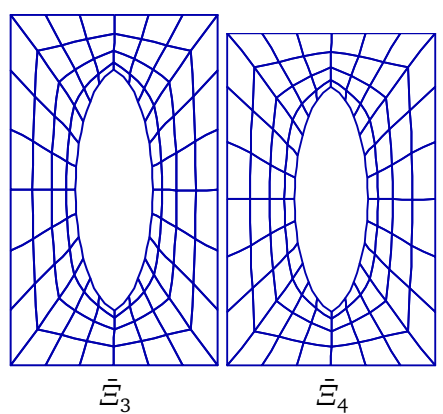

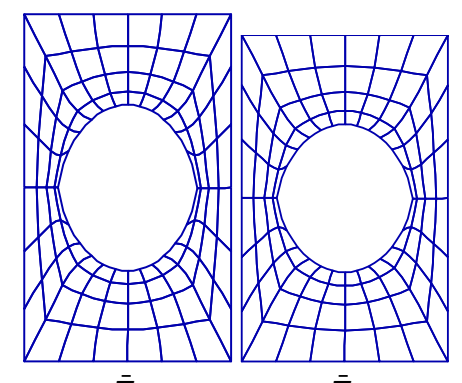

$\bar{\Xi}_{5}$

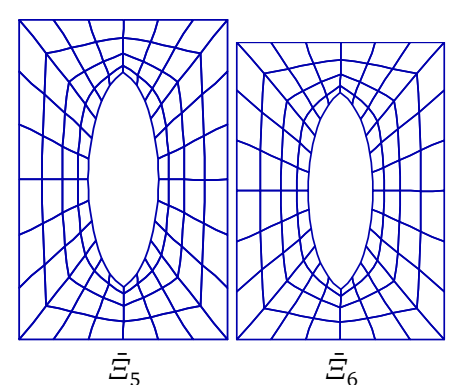

Figure 21: Spatial RVE meshes along the interface at interface integration point coordinates $\bar{\Xi}_{i}=0.522,0.581,0.638,0.737,0.821,0.952$ : (a) horizontally and (b) vertically oriented lens-shaped void. 
(a)

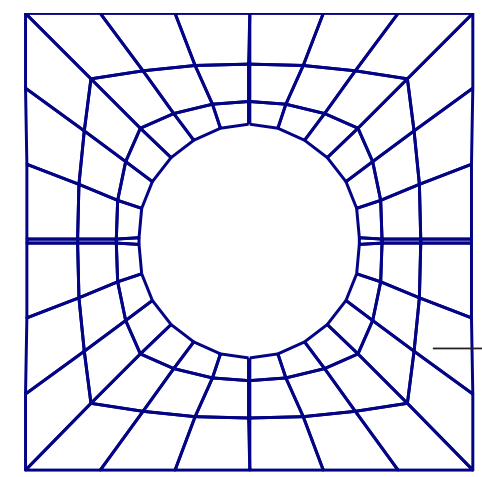

(c)

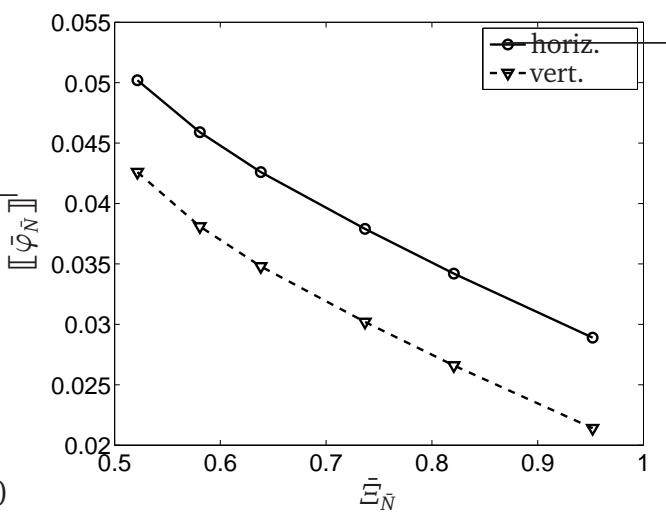

(b)

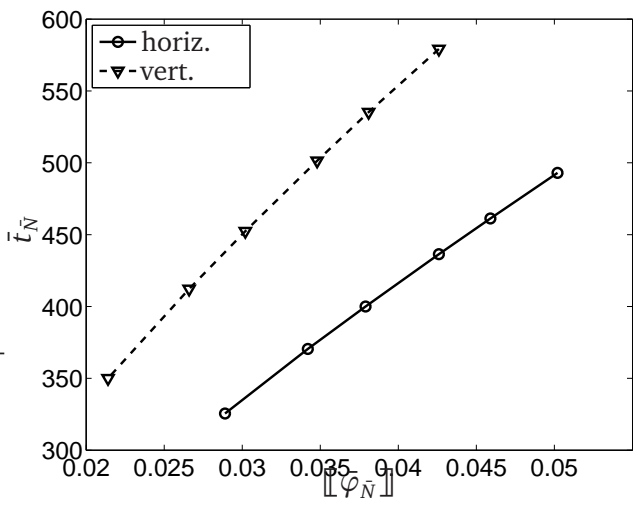

(d)

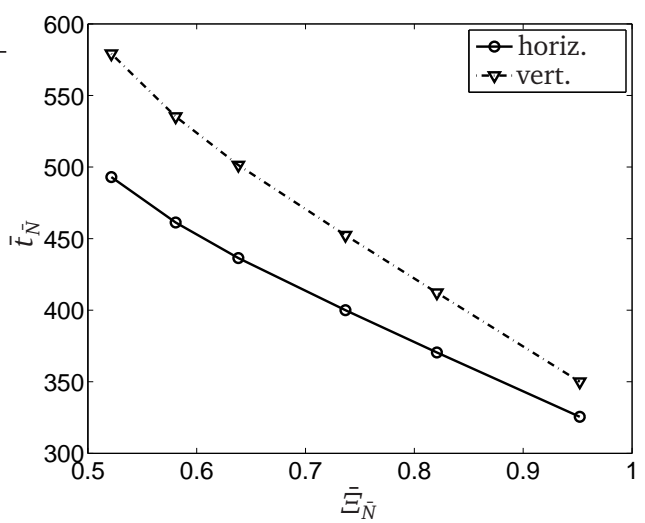

Figure 22: (a) Spatial macro mesh; (b) traction-separation curve at point $\bar{\Xi}=0.522$; (c) separation and (d) traction over the integration point position $\bar{\Xi}$ along the interface. 


\section{Conclusion}

In this contribution, we have proposed a computational homogenization approach for a microscopically heterogeneous material layer. On the basis of the underlying microstructural constitution, the macroscopic response of a body containing this material layer is efficiently determined. The proposed approach is based on a continuum mechanics framework at finite deformations with a cohesive interface to represent the material layer. Based on existing continuum homogenization principles, the vectorial quantities traction and separation at the macro level have been related to the averaged tensorial stress and deformation gradient at the micro level. The height of the representative volume element has been considered as the height of the material layer itself. Thus this quantity enters the equivalence of the virtual work of both scales. The possible tensile and shear deformation modes in the interface have been accounted for through customized boundary conditions on the representative volume element, which are hybrid between prescribed deformation out of the plane and periodic deformation in the plane of the interface. The developed theoretical framework has been successfully embedded in a computational homogenization procedure that couples the macroscopic and the microscopic response in an iterative nested solution procedure. Numerical examples have revealed that the macroscopic response depends on the particular geometry and material properties of the respective microstructure. With the continuous representative volume element and the customized hybrid boundary conditions, mixed-mode loading is captured in a natural manner. Additionally to existing approaches to mixed-mode response, e. g. of References $[29,36]$, the behaviour here is dictated by its microstructure rather than by an a priori constitutive assumption.

Some challenges for future research evolve from this contribution, either concerning the constitutive/continuum formulation or the numerical framework. First of all, in addition to the hyperelastic constitutive format chosen here, the incorporation of irreversible behaviour within the microstructure, as done in Reference [17] for small strain, for finite strains remain as a challenge for future research. Only such choices can render the typical traction-separation laws expected based on References [27, 38]. The present multiscale framework with the classical continuum within the RVE does not account for size effects, which can occur when a significant intrinsic microstructure in the interfacial material coincides with a particularly thin material layer. In such cases, we suggest to employ a generalized continuum as for instance a micromorphic or higher-gradient continuum on the RVE level, as pursued in Reference [7]. One limitation of the multiscale model, which we have particularly identified in the last numerical example, is the fact that although at the macro level there is no obstacle to a lateral contraction of the material layer, the present homogenization framework provides no option to pass this lateral contraction to the underlying representative volume element or vice versa to incorporate the resistance of the RVE against lateral contraction into the homogenization. Recognizing this limitation, it remains as a non-trivial task for future research to enhance the micro-macro transition in this respect such that also a lateral contraction can be taken into account appropriately whenever it is needed.

The interface elements used to model the cohesive layer at the macro level could be 
used in a wider range of boundary value problems, once contact algorithms to capture compression in the interface are implemented. It is noteworthy that the proposed computational homogenization for material layers is not only restricted to finite interface elements, but can be employed whenever a constitutive relation for a cohesive layer is to be evaluated at an integration point. When the proposed computational homogenization framework is combined with more elaborate approaches to treat discontinuous deformations, such as the partition-of-unity based X-FEM [26] or approaches based on Nitsche's method [19], it has potential to serve as a powerful multiscale tool in the simulation of cohesive discontinuities which are governed by their underlying heterogeneous microstructure.

\section{Acknowledgements}

The authors gratefully acknowledge financial support by the German Science Foundation (DFG) within the International Research Training Group 1131 'Visualization of large and unstructured data sets. Applications in geospatial planning, modeling, and engineering' and the Research Training Group 814 'Engineering materials on different scales: Experiment, modelling, and simulation'.

\section{References}

[1] G. Beer. An isoparametric joint/interface element for finite element analysis. Int. J. Numer. Meth. Engng, 21:585-600, 1985.

[2] F. Costanzo, G. L. Gray, and P. C. Andia. On the definitions of effective stress and deformation gradient for use in MD: Hill's macro-homogeneity and the virial theorem. Int. J. Numer. Meth. Engng, 43:533-555, 2005.

[3] F. Feyel and J.-L. Chaboche. $\mathrm{FE}^{2}$ multiscale approach for modelling the elastoviscoplastic behaviour of long fibre SiC/Ti composites materials. Comput. Methods Appl. Mech. Engrg., 183:309-330, 2000.

[4] R. Hill. Elastic properties of reinforced solids: Some theoretical principles. J. Mech. Phys. Solid., 11:357-372, 1963.

[5] R. Hill. On constitutive macro-variables for heterogeneous solids at finite strain. Proc. Roy. Soc. Lond. A, 326:131-147, 1972.

[6] C. B. Hirschberger. A Treatise on Micromorphic Continua. Theory, Homogenization, Computation. PhD thesis, University of Kaiserslautern, 2008. ISSN 1610-4641, ISBN 978-3939432-80-7.

[7] C. B. Hirschberger, N. Sukumar, and P. Steinmann. Computational homogenization of material layers with micromorphic mesostructure. Phil. Mag., accepted, 2008.

[8] V. G. Kouznetsova. Computational Homogenization for the Multiscale Analysis of Multi-Phase Materials. PhD thesis, Eindhoven University of Technology, 2002. 
[9] V. G. Kouznetsova, W. A. M. Brekelmans, and F. P. T. Baaijens. An approach to micro-macro modeling of heterogeneous materials. Comput. Mech., 27:37-48, 2001.

[10] V. G. Kouznetsova, M. G. D. Geers, and W. A. M. Brekelmans. Multi-scale constitutive modelling of heterogeneous materials with a gradient-enhanced computational homogenization scheme. Int. J. Numer. Meth. Engng, 54:1235-1260, 2002.

[11] V. G. Kouznetsova, M. G. D. Geers, and W. A. M. Brekelmans. Multi-scale second-order computational homogenization of multi-phase materials: a nested finite element solution strategy. Comput. Methods Appl. Mech. Engrg., 193:5525-5550, 2004.

[12] R. Larsson, K. Runesson, and N. S. Ottosen. Discontinuous displacement approximation for capturing plastic localization. Int. J. Numer. Meth. Engng, 36:2087-2105, 1993.

[13] R. Larsson, K. Runesson, and S. Sture. Finite element simulation of localized plastic deformation. Arch. Appl. Mech., 61:305-317, 1991.

[14] R. Larsson and Y. Zhang. Homogenization of microsystem interconnects based on micropolar theory and discontinuous kinematics. J. Mech. Phys. Solid., 55:819-841, 2007.

[15] F. Lebon, A. Ould Khaoua, and C. Licht. Numerical study of soft adhesively bonded joints in finite elasticity. Comput. Mech., 21:134-140, 1998.

[16] F. Lebon, R. Rizzoni, and S. Ronel-Idrissi. Asymptotic analysis of some non-linear soft layers. Comput. Struct., 82:1929-1938, 2004.

[17] K. Matous, M. G. Kulkarni, and P. H. Geubelle. Multiscale cohesive failure modeling of heterogeneous adhesives. J. Mech. Phys. Solid., 56:1511-1533, 2008.

[18] J. Mergheim, E. Kuhl, and P. Steinmann. A hybrid discontinuous Galerkin/interface method for the computational modelling of failure. Commun. Numer. Meth. Engng, 20:511-519, 2004.

[19] J. Mergheim, E. Kuhl, and P. Steinmann. A finite element method for the computational modelling of cohesive cracks. Int. J. Numer. Meth. Engng, 63:276-289, 2005.

[20] J. C. Michel, H. Moulinec, and P. Suquet. Effective properties of composite materials with periodic microstructure: a computational approach. Comput. Methods Appl. Mech. Engrg., 172:109-143, 1999.

[21] C. Miehe. Computational micro-to-macro transitions discretized micro-structures of heterogeneous materials at finite strains based on the minimization of averaged incremental energy. Comput. Methods Appl. Mech. Engrg., 192:559-591, 2003.

[22] C. Miehe and A. Koch. Computational micro-to-macro transitions of discretized microstructures undergoing small strains. Arch. Appl. Mech., 72:300-317, 2002.

[23] C. Miehe and J. Schröder. Post-critical discontinuous localization analysis of small-strain softening elastoplastic solids. Arch. Appl. Mech., 64:267-285, 1994.

[24] C. Miehe, J. Schröder, and C. Bayreuther. On the homogenization analysis of composite materials based on discretized fluctuations on the micro-structure. Comput. Mater. Sci., 155:1-16, 2002. 
[25] C. Miehe, J. Schröder, and J. Schotte. Computational homogenization analysis in finite plasticity. Simulation of texture development in polycrystalline materials. Comput. Methods Appl. Mech. Engrg., 171:387-418, 1999.

[26] N. Moës, J. Dolbow, and T. Belytschko. A finite element method for crack growth without remeshing. International Journal for Numerical Methods in Engineering, 46:131-150, 1999.

[27] A. Needleman. A continuum model for void nucleation by inclusion debonding. J. Appl. Mech., 54:525-531, 1987.

[28] A. Nemat-Nasser and M. Hori. Micromechanics: Overall Properties of Heterogeneous Materials. North-Holland Elsevier, 2nd revised edition, 1999.

[29] M. Ortiz and A. Pandolfi. Finite-deformation irreversible cohesive elements for threedimensional crack-propagation analysis. Int. J. Numer. Meth. Engng, 44:1267-1282, 1999.

[30] J. C. J. Schellekens and R. de Borst. On the numerical integration of interface elements. Int. J. Numer. Meth. Engng, 36:43-66, 1993.

[31] P. Steinmann. A model adaptive strategy to capture strong discontinuities at large inelastic strain. In S. Idelsohn, E. Oñate, and E. Dvorking, editors, Computational Mechanics. New Trends and Applications, pages 1-12. CIMNE, Barcelona, 1998.

[32] P. Steinmann and P. Betsch. A localization capturing FE-interface based on regularized strong discontinuities at large inelastic strains. Int. J. Solid Struct., 37:4061-4082, 2000.

[33] N. Sukumar and J. E. Pask. Classical and enriched finite element formulations for Blochperiodic boundary conditions. Int. J. Numer. Meth. Engng, 2008. DOI: 10.1002/nme.2457.

[34] J. Utzinger, M. Bos, M. Floeck, A. Menzel, E. Kuhl, R. Renz, K. Friedrich, A.K. Schlarb, and P. Steinmann. Computational modelling of thermal impact welded PEEK/steel single lap tensile specimens. Comput. Mater. Sci., 41(3):287-296, 2008.

[35] J. Utzinger, A. Menzel, P. Steinmann, and A. Benallal. Aspects of bifurcation in an isotropic elastic continuum with orthotropic inelastic interface. Eur. J. Mech. A Solid, 27:532-547, 2008.

[36] M. J. van den Bosch, P. J. G. Schreurs, and M. G. D. Geers. An improved description of the exponential Xu and Needleman cohesive zone law for mixed-mode decohesion. Eng. Fract. Mech., 73:1220-1234, 2006.

[37] M. J. van den Bosch, P. J. G. Schreurs, and M. G. D. Geers. On the development of a 3d cohesive zone element in the presence of large deformations. Comput. Mech., 42:171-180, 2008.

[38] X.-P. Xu and A. Needleman. Void nucleation by inclusion debonding in a crystal matrix. Modelling Simul. Mater. Sci. Eng., 1:111-132, 1993. 